\title{
Article
}

\section{Transcriptomic Analysis Reveals Differential Expression of Genes between Lung Capillary and Post Capillary Venules in Abdominal Sepsis}

\author{
Milladur Rahman ${ }^{+}\left(\mathbb{D}\right.$, Zhiyi Ding ${ }^{\dagger}$, Carl-Fredrik Rönnow (D) and Henrik Thorlacius *
}

check for

updates

Citation: Rahman, M.; Ding, Z.; Rönnow, C.-F.; Thorlacius, H.

Transcriptomic Analysis Reveals Differential Expression of Genes between Lung Capillary and Post Capillary Venules in Abdominal Sepsis. Int. J. Mol. Sci. 2021, 22, 10181. https://doi.org/10.3390/ ijms221910181

Academic Editor: Ignacio Benedicto

Received: 29 August 2021

Accepted: 17 September 2021

Published: 22 September 2021

Publisher's Note: MDPI stays neutral with regard to jurisdictional claims in published maps and institutional affiliations.

Copyright: (c) 2021 by the authors. Licensee MDPI, Basel, Switzerland. This article is an open access article distributed under the terms and conditions of the Creative Commons Attribution (CC BY) license (https:// creativecommons.org/licenses/by/ $4.0 /)$.
Department of Clinical Sciences, Malmö, Section for Surgery, Lund University, 21428 Malmö, Sweden; milladur.rahman@med.lu.se (M.R.); zhiyi.ding.3635@med.lu.se (Z.D.); carl-fredrik.ronnow@med.lu.se (C.-F.R.)

* Correspondence: henrik.thorlacius@med.lu.se

+ Equal contributor.

Abstract: Lung endothelial cell dysfunction plays a central role in septic-induced lung injury. We hypothesized that endothelial cell subsets, capillary endothelial cells (capEC) and post capillary venules (PCV), might play different roles in regulating important pathophysiology in sepsis. In order to reveal global transcriptomic changes in endothelial cell subsets during sepsis, we induced sepsis in C57BL/ 6 mice by cecal ligation and puncture (CLP). We confirmed that CLP induced systemic and lung inflammation in our model. Endothelial cells (ECs) from lung capillary and PCV were isolated by cell sorting and transcriptomic changes were analyzed by bioinformatic tools. Our analysis revealed that lung capEC are transcriptionally different than PCV. Comparison of top differentially expressed genes (DEGs) of capEC and PCV revealed that capEC responses are different than PCV during sepsis. It was found that capEC are more enriched with genes related to regulation of coagulation, vascular permeability, wound healing and lipid metabolic processes after sepsis. In contrast, PCV are more enriched with genes related to chemotaxis, cell-cell adhesion by integrins, chemokine biosynthesis, regulation of actin filament process and neutrophil homeostasis after sepsis. In addition, we predicted some transcription factor targets that regulate a significant number of DEGs in sepsis. We proposed that targeting certain DEGs or transcriptional factors would be useful in protecting against sepsis-induced lung damage.

Keywords: endothelial cells; lung; RNAseq; inflammation; sepsis

\section{Introduction}

Sepsis is a dominating cause of hospital-related mortality all over the world [1]. The management of sepsis patients is largely confined to supportive care which is partly due to an incomplete understanding of the pathophysiology [2]. The annual cost for sepsis is EUR 7.6 billion in Europe [2] and USD 24 billion in USA [3]. The substantial costs and lack of effective therapies in sepsis have urged a more thorough understanding of immune pathology of sepsis in order to develop therapies that will significantly modulate the outcome of the disease. Sepsis usually leads to single organ dysfunction, typically acute lung injury, followed by multiple organ dysfunctions [4]. Lung dysfunction is considered to be an insidious part of sepsis. Under resting conditions, endothelial cells (EC) play a pivotal part in maintaining normal homeostasis. However, during sepsis almost all of the aspects of EC function, including barrier function, vasoregulation and homeostasis, become dysregulated [5-7]. One pathophysiological hallmark of sepsis is excessive tissue infiltration of leukocytes and disruption of gaseous exchange [6,7]. We and others have previously shown that leukocyte recruitment into the lung tissue is a multistep process, involving tethering, rolling, firm adhesion and extravascular migration, mediated by specific adhesion molecules expressed on leukocytes and ECs [8,9]. For example, 
the selectin family of adhesion molecules, including P, E- and L-selectin, supports leukocyte tethering and rolling on vascular ECs in an organ-specific manner [10].

Another hallmark of sepsis is dysfunctional coagulation. It is well known that sepsisinduced mortality is greatly enhanced in the presence of disseminated intravascular coagulation [11]. During inflammation, the vascular endothelium becomes procoagulant and prothrombotic, causing excessive coagulation leading to microcirculatory tissue perfusion failure and organ damage [12,13]. Several therapies targeting EC function, such as recombinant human tissue factor pathway inhibitor, recombinant-activated protein $\mathrm{C}$, recombinant-soluble thrombomodulin, protein $\mathrm{C}$ concentrate and heparin have been tested in previous trials, with no significant effect on clinical outcome $[5,14]$. A possible explanation to this could be that sepsis manifests itself into multiple processes, making the condition difficult to treat by targeting a single process. Additionally, concerns have been raised on the rapid dissemination of knowledge originating from lethal toxin-based animal studies to clinical trials without investigating the pathophysiological aspects further in experimental sepsis models. It is our expectation that a global analysis of adhesion molecules, cytokines and coagulation factors, as well as identification of transcriptional factors involved in dysregulation of EC, will provide unique insight in the chain reactions of sepsis, facilitating the next generation therapeutic regimens in sepsis.

The microvascular bed is composed of small arterioles, capillaries and postcapillary venules with a diameter less than $50 \mu \mathrm{m}$ [15]. Early studies revealed that there are fundamental structural variations between microvascular beds in different organs primarily in microvascular patterns and capillary ultrastructure. For example, capillaries are fenestrated in the liver and kidney but not in the lung, heart and brain [16]. In the present study, we intended to investigate transcriptional changes and regulation in terms of inflammation, barrier function and coagulation in capEC and PCV of lung microvasculature, in an experimental model of sepsis. Our analysis revealed different transcriptomic changes in specific lung-derived EC and predicted some transcriptional factors that could be targeted in order to protect against sepsis-induced lung damage and dysfunction.

\section{Results}

\subsection{Lung capEC and PCV Are Transcriptionally Different}

For transcriptomic analysis of lung EC subsets, we sorted EC subsets using a modified protocol [17] described details in the methods section. We sorted blood capillary and post capillary venular ECs through the use of monoclonal antibodies. The capEC subset was identified as CD31+Icam1+Vcam1- and PCV subset was identified as CD31+Icam1+Vcam1+ (Figure 1A). The identity of capEC and PCV were further confirmed by two other antibody panels where capEC was identified as CD31+Icam1+CD63- and CD31+Ly6c+Vcam1-; PCV was identified as CD31+Icam1+CD63+ and CD31+Ly6c+Vcam1+ (Figure 1B).

Flow cytometry analysis revealed that $\mathrm{CD} 63$, a tetraspanin protein linked to leukocyte adhesion and recruitment [18], was low on capEC but high on PCV. Icam1 is known to be expressed to a high degree in capEC and to a low degree in arteries [19]. In peripheral lymph nodes and peyer's patch, Vcam1 was found to be highly expressed in post capillary venules (known as HECs) but very low or undetectable in capEC [17]. By flow cytometric analysis, we found that lung BECs uniformly expressed Icam1 and Ly6c and use of Vcam1 and CD63 antibodies together with Icam1 or Ly6c antibodies separate lung capEC from PCV. capEC and PCV separation was better using Icam1 and Vcam1 markers than any other combinations, therefore, we sorted ECs (CD31+gp38-) with high expression of Icam1 and low expression for Vcam1 as capEC and ECs with high expression of Vcam1 and Icam 1 as PCV. The purity of the sorted capEC was confirmed to be $97.5 \%$ (Supplementary Figure S1). Gene expression analysis of sham capEC and PCV revealed that ECs sorted as capEC expressed capillary-specific signature genes, including CD36, Nes, Hey1 and Notch4 (Figure 1C). On the other hand, BECs sorted as PCV expressed venule-specific signature genes, including CD63, Bst1, Tlr4 and Nr2f2 (Figure 1C), suggesting that sorted capEC and PCV are transcriptionally different as observed by Lee M, et al. [17]. Principal 
component analysis (PCA) showed that biological replicates of capEC and PCV cluster together (Supplementary Figure S2A), suggesting that samples in capEC have similar expression but different than PCV group. Top 100 DEGs of sham capEC and PCV were displayed in a PCA plot (Supplementary Figure S2B) $\left(p \leq 1.9 \times 10^{-15}\right.$, padj $\leq 2.9 \times 10^{-13}$; $>4$-fold difference). Top 100 DEGs in sham capEC and PCV in a PCA plot also suggested that lung capEC and PCV are transcriptionally different (Supplementary Figure S2C). For example, capEC were found to be enriched with genes involved in cell proliferation and angiogenesis, such as Dpp4, Nes, Kitl and Kit (Supplementary Figure S2C). In contrast, PCV were found to be enriched with genes involved in cell adhesion and migration, such as Bst1, ptk2b, Vwf, Amigo2, Itgb3, Itgb4 and Cdh13 (Supplementary Figure S2C). PCV were also found to be enriched with master venous regulator $N r 2 f 2$ in the top 100 genes.

A
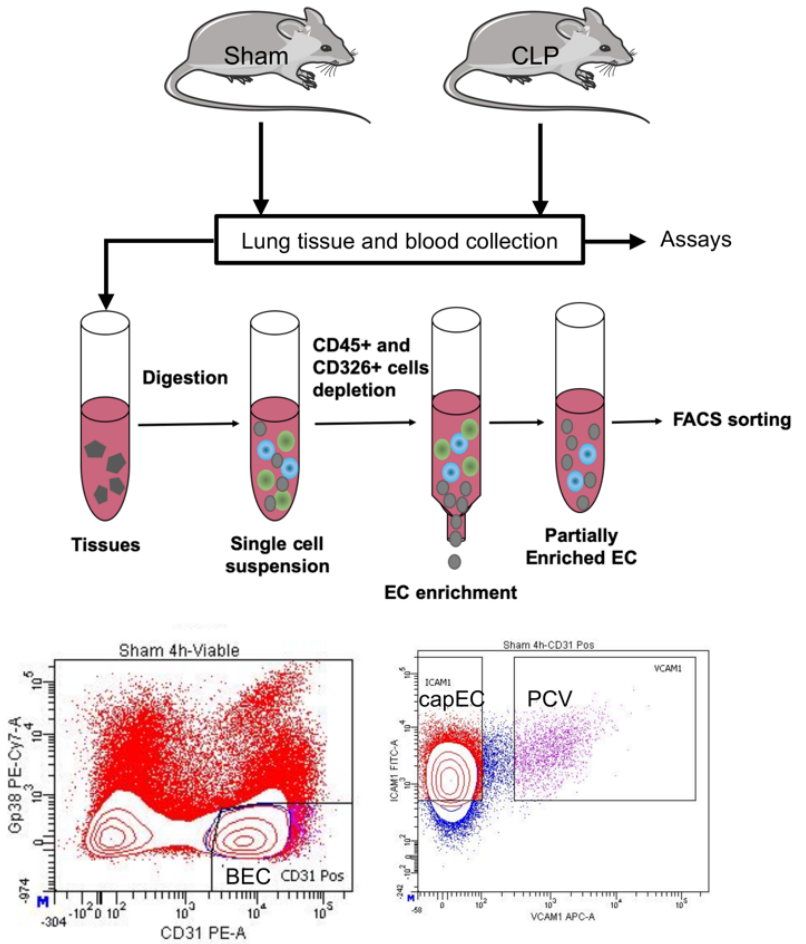

B
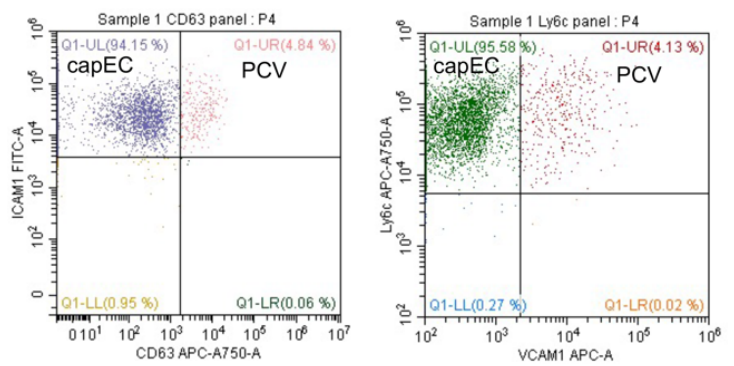

C Capillary-specific genes

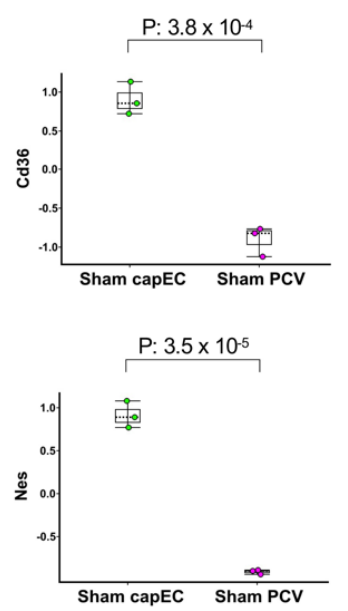

P: $1.3 \times 10^{-2}$
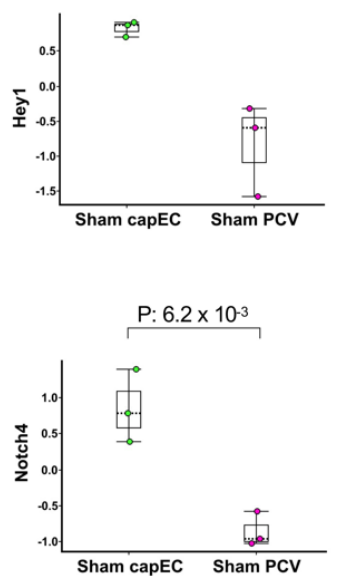

Venular-specific genes
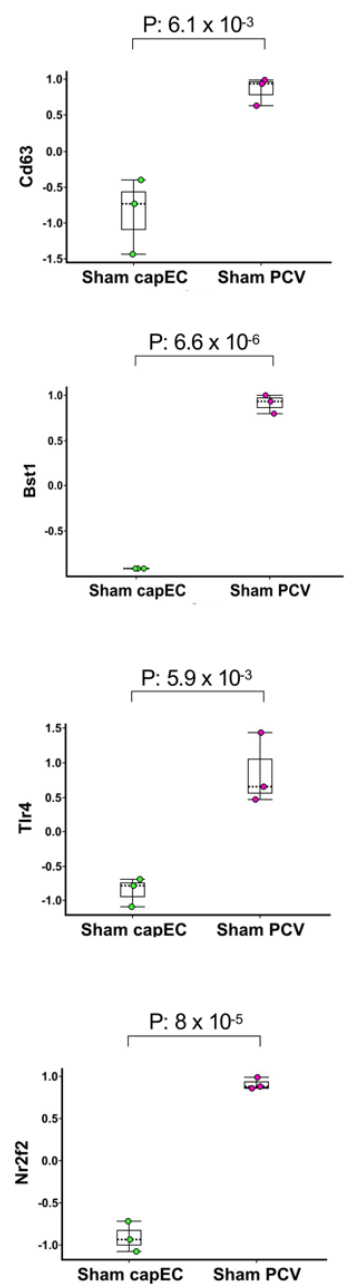

Figure 1. Lung endothelial subsets isolation and transcriptional signature genes. (A) Upper panel showing experimental design; middle panel showing lung tissue digestion and EC enrichment by depletion of CD45+ and CD326+ cells; lower panel showing flow cytometry gating strategy for the isolation of capEC and PCV from CD31+gp38- blood ECs (BECs) of lungs through the use of antibodies against Icam1 and Vcam1. (B) Identity of blood capEC and PCV was further confirmed by flow cytometry using Ly6c and venular-specific CD63 markers. (C) Expression of capillary-specific signature genes (Cd36, Nes, Hey1 and Notch4) in CD31+Icam1+Vcam1- BECs and venular-specific signature genes (Cd63, Vcam1, Tlr4 and Nr2f2) in CD31+Icam1+Vcam1+ BECs. Samples were collected $4 \mathrm{~h}$ after sham operation. Box plots represent the median (25-75 percentile) and the whiskers extend from the minimum to the maximum levels and dots represent sample values, normalized to mean $=0$, variance $=1$. $p$-value indicates statistical significance level. 


\subsection{Sepsis Induces Pulmonary and Systemic Inflammation}

To confirm pulmonary and systemic inflammation $4 \mathrm{~h}$ after CLP, we performed biochemical and immunological assays. MPO (an indicator of neutrophils) and Mac1 expression on the surface of circulating neutrophils were examined by enzymatic assay and flow cytometry, respectively. We observed that pulmonary MPO activity increased by more than 3-fold in CLP animals (Figure 2A). Flow cytometry analysis revealed that Mac1 expression on circulating neutrophils increased by more than 6-fold in CLP animals (Figure 2B). Moreover, CLP markedly increased pulmonary and plasma formation of Cxcl2 and Il6 (Figure 2C-F), suggesting that $4 \mathrm{~h}$ CLP indeed caused clear-cut pulmonary and systemic inflammation.

A

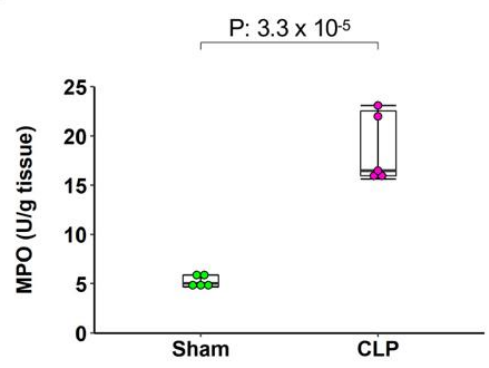

B

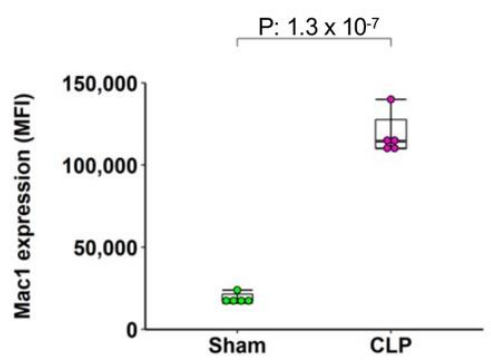

C

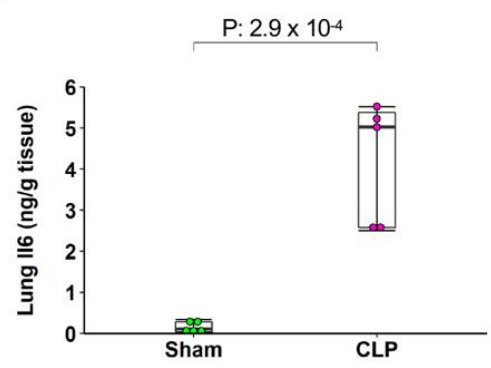

D

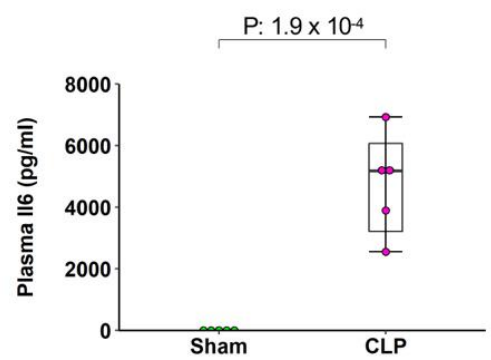

$E$

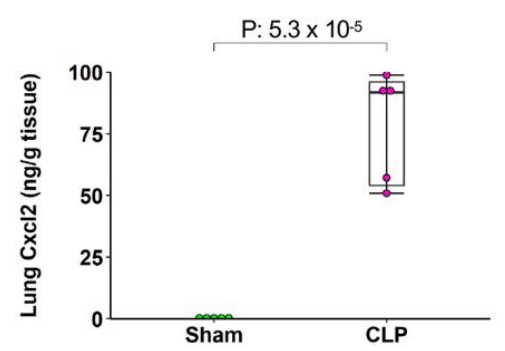

$\mathrm{F}$

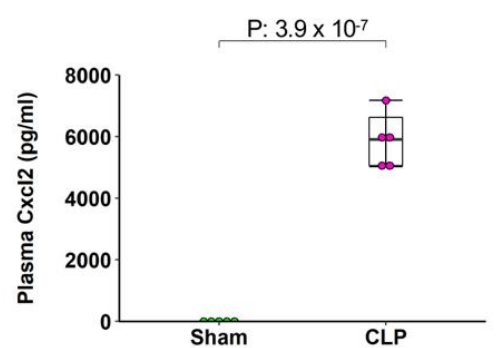

Figure 2. Tissue and systemic inflammation after induction of abdominal sepsis. (A) Pulmonary MPO; (B) Il6; (C) Cxcl2; (D) Mac1 expression on circulatory neutrophils; (E) plasma Il6 and (F) plasma Cxcl2. Samples were collected $4 \mathrm{~h}$ after sham and CLP. Box plots represent the median (25-75 percentile) and the whiskers extend from the minimum to the maximum levels and dots represent sample values and $n=5$. $p$-value indicates statistical significance level.

\subsection{Differential Gene Expression between capEC and PCV during Sepsis}

Our analysis revealed that, lung EC subsets had differential expression of genes involved in different types of biological functions. In the top 100 DEGs of capEC (sham vs. CLP), we observed expression of genes related to cellular response to lipopolysaccharide (Cebpb, Cd14, Litaf, Adam9, Acod1, Il6, Icam19), protein phosphorylation (Nuak2, Riok3, Smad1, Ccl3, Map2k3, Pim3, Syk), apoptotic process (Litaf, Nuak2, Birc3, Tia1, Shb, Tnfaip3), blood vessel morphogenesis (Shb, Thbs1, Syk) and positive regulation of cell extravasation (Adam8, Icam1, Ptafr) (Figure 3A and Supplementary Material S1). In contrast, top 100 DEGs of PCV (sham vs. CLP), expressed genes linked to cellular response to lipopolysaccharide (Wfdc21, Cxcl1, Il10ra, Irak3, Noct, Sod2, Trib1, Litaf), acute-phase response (Il6, Serpina3n, Saa3), positive regulation of tumor necrosis factor production (Ccl4, Ptafr, Tlr2), cytokine-mediated cell signaling (Illfa, Il6, Irak3, Socs2), response to hypoxia (Fosl2, Hif1a, Sod2, Tlr2) and neutrophil chemotaxis (Amica1, ccl4, Cxcl1) (Figure 3B and Supplementary Material S2). We compared the top 100 DEGs between capEC (from sham vs. CLP) and PCV (from sham vs. CLP) and found that gene expression between them was different. It was observed that in the top 100 DEGs, capEC and PCV had 37 genes in common (Adam9, Adamts9, Apobr, Bcl3, Birc3, Cxcl1, Cyp26b1, D10Wsu102e, Dennd4a, Dusp16, Gramd1a, Il6, Litaf, Map3k8, Noct, 
Nuak2, Osmr, Pfkfb3, Ptafr, Samsn1, Sema4c, Serpina3f, Serpina3g, Smad1, Smad7, Snx10, Socs2, Sod2, Stx11, Susd6, Thbs1, Tlr2, Tnfaip3, Tnip1, Trib1, Wfdc21, Zfp46) mainly involved in immune response and apoptosis, whilst 84 genes differed (capEC left panel, PCV right panel, Figure 3C). PCA analysis showed that biological replicates of sham capEC cluster together, same for biological replicates of CLP capEC, sham PCV and CLP PCV (Supplementary Figure S3). The first principal component (largest difference) separates Sham PCV from CLP PCV, indicating that CLP caused massive transcriptional changes in PCV (Supplementary Figure S3E). Second principal component separates capEC from $\mathrm{PCV}$, indicating endothelial subsets specific transcriptional differences. Third principal component (lowest difference) separates sham capEC from CLP capEC, indicating CLP caused relatively less transcriptional changes in capEC (Supplementary Figure S3E). We also compared the top 500 genes and found only 185 common genes between capEC and PCV DEGs (not shown), confirming that CLP caused distinct transcriptional changes in capEC and PCV.

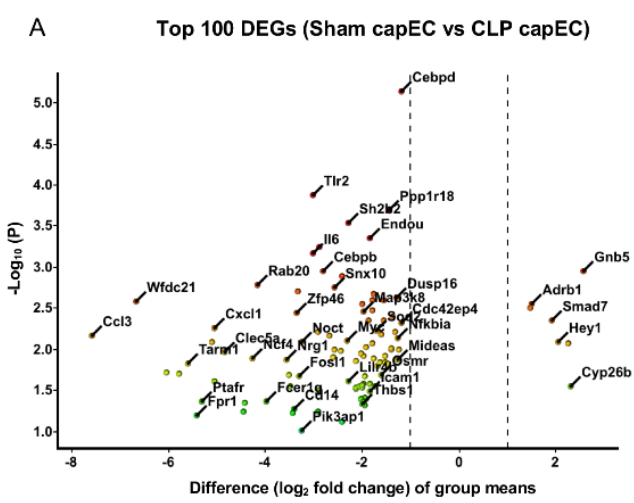

B Top 100 DEGs (Sham PCV vs CLP PCV)
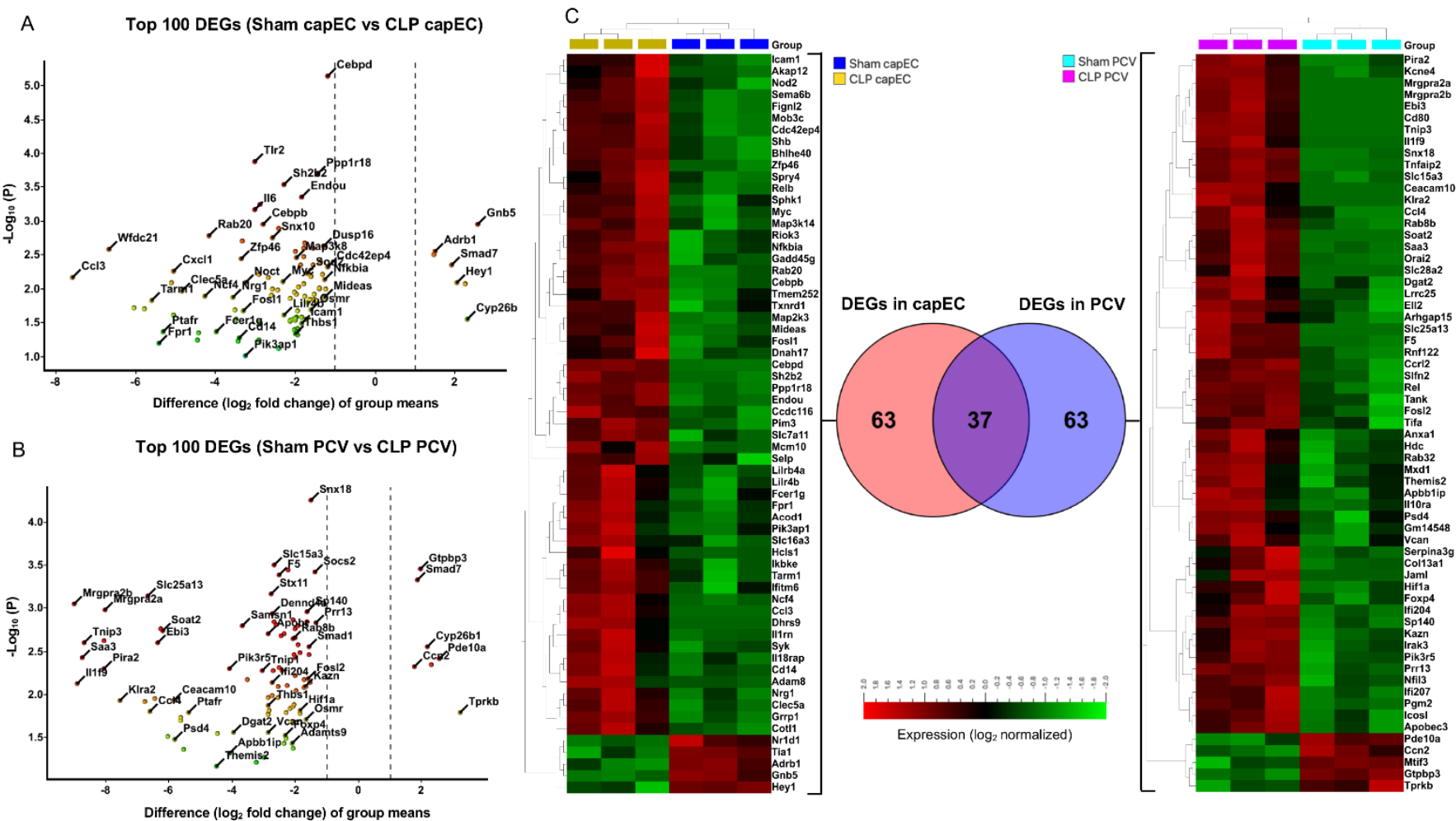

Figure 3. Top 100 differentially expressed genes (DEGs) in lung EC subsets. (A) Volcano plot showing top 100 DEGs in the capEC (sham capEC vs. CLP capEC), $p \leq 3.4 \times 10^{-6}$, padj $\leq 0.0004$. (B) Volcano plot showing top 100 DEGs in the PCV (Sham PCV vs. CLP PCV), $p \leq 1.9 \times 10^{-6}, \mathrm{q} \leq 0.0002$. Volcano plot is constructed by plotting the negative $\log 10$ of the $p$ value versus the group mean difference ( $\log 2$ fold change). Variables are colored based on fold change, blue dots indicate upregulated in CLP and red dots indicate downregulated n CLP. Highly differed variables are in the top left and top right areas of the volcano plot. (C) Venn diagram showing overlapping of top 100 DEGs between capEC and PCV (middle panel), 84 different variables in capEC are shown in left heatmap and 84 different variables in PCV are shown in right heat map. Hierarchical clustering and box color of heat map are based on data normalization, mean $=0$, variance $=1$. $p$-value indicates statistical significance level and q-value indicates false discovery rate.

\subsection{Distinct Gene Sets and Pathways Enrichment between capEC and PCV}

Next, we performed Gene Set Enrichment Analysis (GSEA) using predefined sets of genes based on functional annotation of DEGs. Our analysis revealed discordance between capEC (sham vs. CLP) and PCV (sham vs. CLP). We selected and compared the most significantly enriched signaling pathway, based on the Normalized Enrichment Score (NES) for capEC and PCV, respectively (Figure $4 \mathrm{~A}$ ). Our analysis revealed that sepsis caused 
higher gene sets enrichment in capEC compared with PCV in the following GO terms: adherens junction organization, angiogenesis, GDP metabolic process, glycoside catabolic process, insulin metabolic process, lipid metabolic process, notch signaling pathway, Wnt signaling pathway, VEGF signaling, plasma membrane tubulation, regulation of coagulation, regulation of vascular permeability and wound healing involved in inflammatory response (Figure 4A). Concurrently, sepsis caused higher gene sets enrichment in PCV in the following GO terms: cell chemotaxis, cell-cell adhesion by cadherins, cell-cell adhesion by integrins, chemokine biosynthesis process, calcium-mediated signaling, negative regulation of blood circulation, negative regulation of plasminogen activation, negative regulation of sodium ion transport, regulation of actin filament process, neutrophil homeostasis and response to prostaglandin (Figure 4A). Three selected gene sets from capEC (Figure 4B) and three from PCV (Figure 4C) are presented to show running enrichment score (RES). Selected DEGs of capEC based on relevant GO terms (Figure 5) and selected DEGs of PCV based on relevant GO terms (Figure 6), were presented as heatmaps.

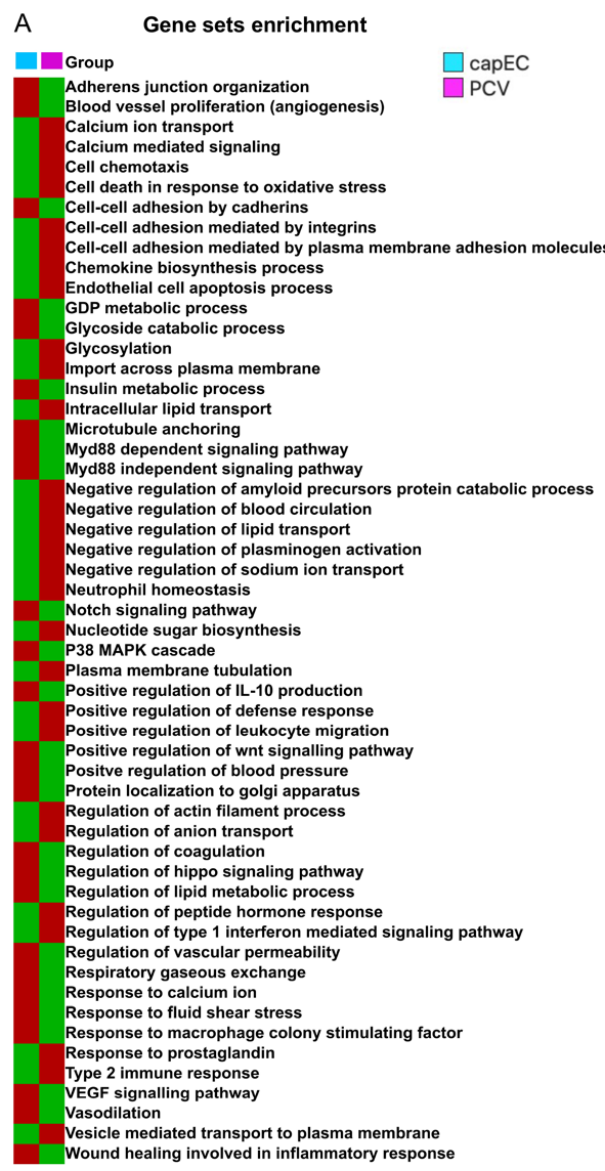

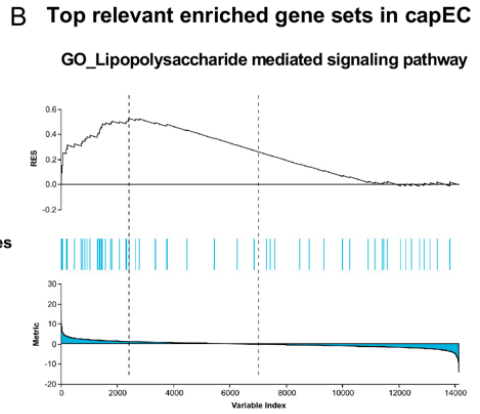

GO_VEGF receptor signaling pathway

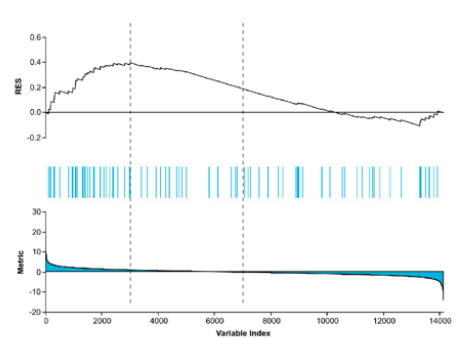

GO_Regulation of coagulation

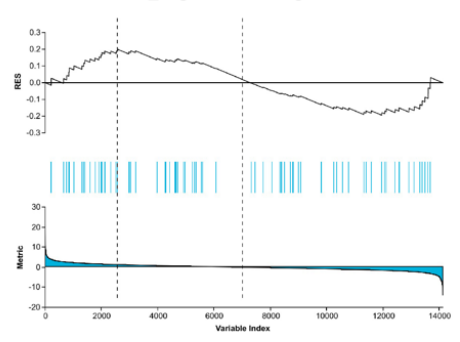

C Top relevant enriched gene sets in PCV

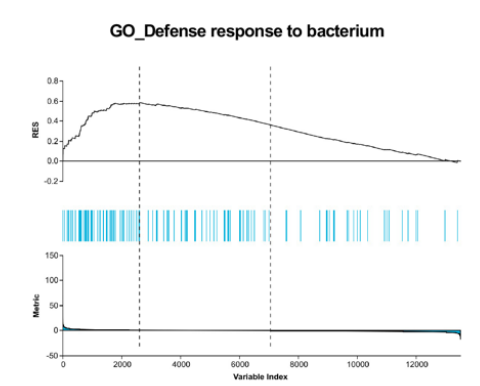

GO_Positive regulation of Chemokine production

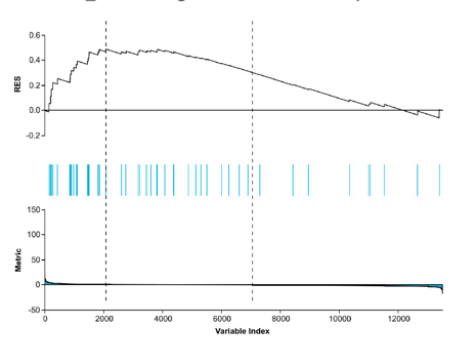

GO_Cell-cell adhesion via plasma membrane adhesion molecules

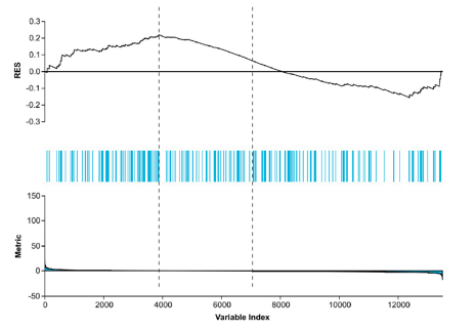

Figure 4. Gene sets enrichment analysis. (A) Heat map showing differentially enriched GO pathways based on normalized enrichment score in lung capEC and PCV after induction of sepsis, red indicates higher gene sets enrichment and green indicates lower gene sets enrichment. (B) Top three relevant enriched gene sets (human orthologs) in lung capEC. (C) Top three relevant enriched gene sets (human orthologs) in lung PCV. For GSEA analysis, mouse genes are converted to human orthologs as described in the materials and methods section. GSEA analysis was performed using gene sets derived from the GO Biological Process (BP) ontology of the Molecular Signatures Database (v7.2 MSigDB). Plots show running enrichment score (RES), the match positions for the genes (blue lines) in the selected data set and the ranking metric values for the input gene list. 
Heat maps showing DEGs in capEC based on enriched GO terms
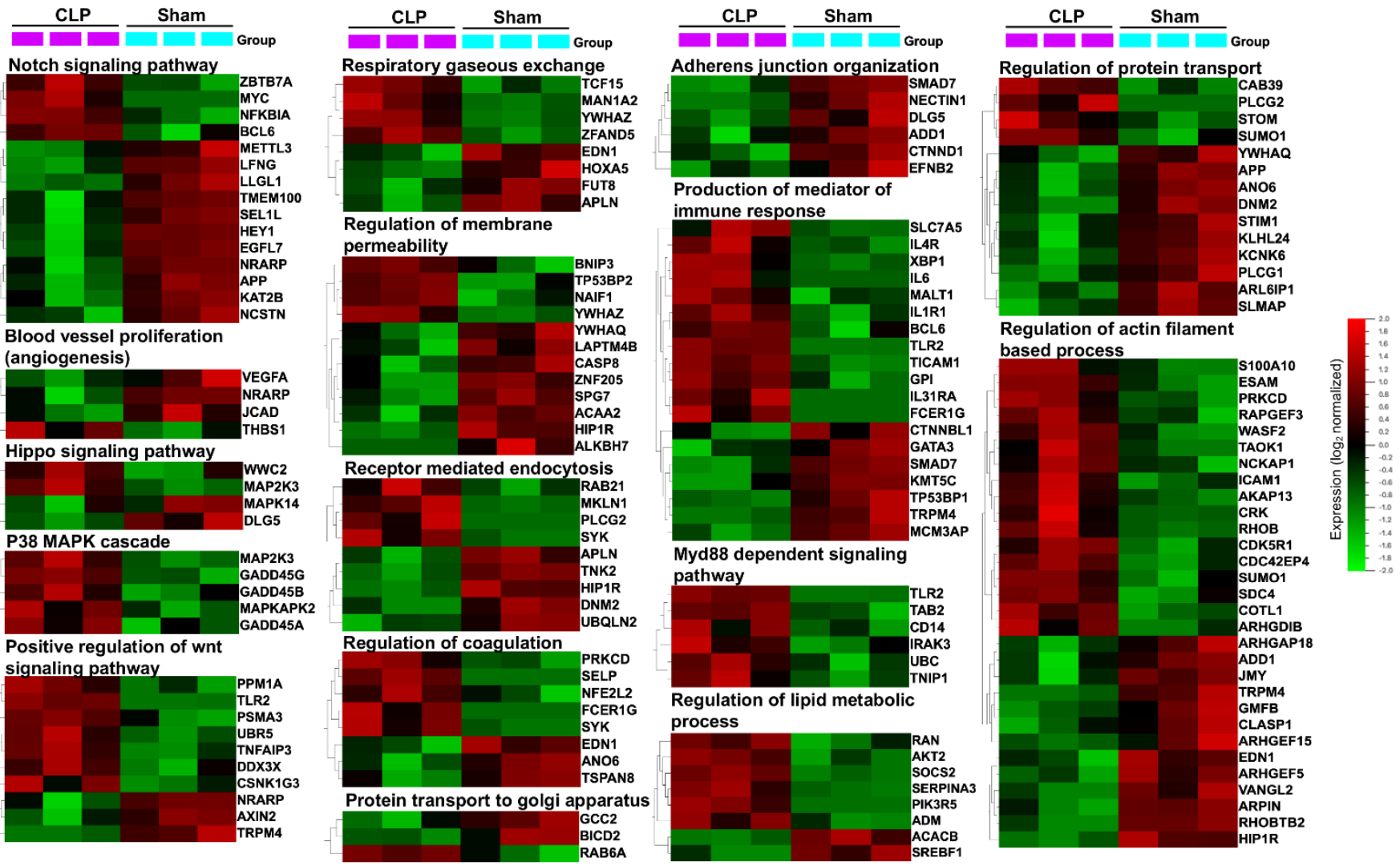

Figure 5. Heat maps showing DEGs $(p \leq 0.05)$ with GO enriched term in capEC. Samples were collected 4 h after induction of abdominal sepsis. GO terms were based on biological process of Molecular Signatures Database (v7.2 MSigDB). For GSEA analysis, mouse genes were converted to human orthologs as described in the materials and methods section. Hierarchical clustering of variables and box color of heat maps were based on data normalization, mean $=0$, variance $=1$.

KEGG pathway enrichment analyses using the top 500 DEGs revealed that both capEC and PCV have similar types of pathway enrichment (Tables 1 and 2). In the DEGs of capEC, the most enriched pathway was TNF signaling pathway (Top upregulated TNF signalingrelated genes were shown in Supplementary Figure S4), followed by NOD-like receptor signaling pathway, Toll-like receptor signaling pathway, apoptosis pathway, NF-kappa B signaling pathway, cytokine-cytokine receptor interaction, MAPK signaling pathway, p53 signaling pathway, TGF-beta signaling pathway and chemokine signaling pathway. In the DEGs of PCV, the most enriched pathway was TNF signaling pathway, followed by NF-kappa B signaling pathway (top upregulated NF-kappa B signaling related genes are shown in Supplementary Figure S5), Toll-like receptor signaling pathway, NOD-like receptor signaling pathway, cytokine-cytokine receptor interaction, apoptosis, cell adhesion molecules, JAK-STAT signaling pathway, HIF-1 signaling pathway and TGF-beta signaling pathway. 
Heat maps showing DEGs in PCV based on enriched GO terms
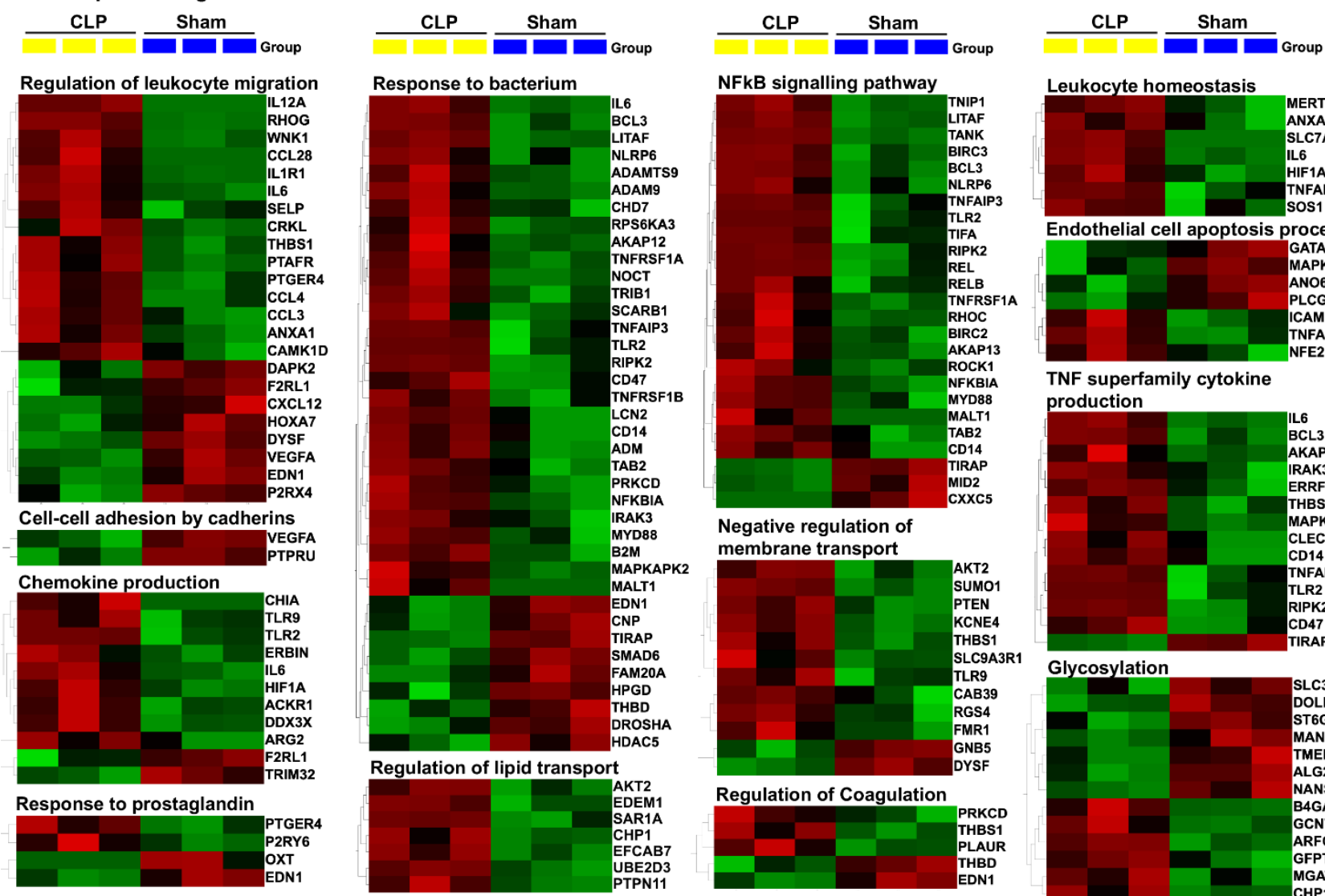

Leukocyte homeostasis
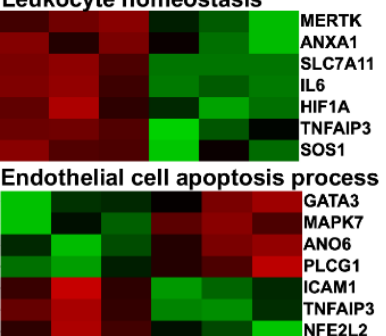

TNF superfamily cytokine production

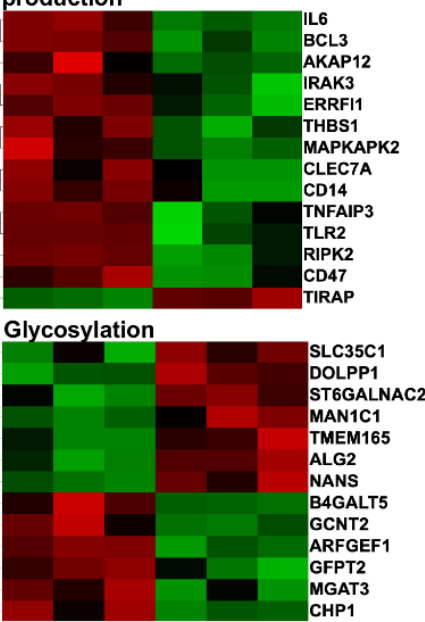

Figure 6. Heat maps showing DEGs $(p \leq 0.05)$ with GO enriched term in PCV. Samples were collected 4 h after induction of abdominal sepsis. GO terms were based on biological process of Molecular Signatures Database (v7.2 MSigDB). For KEGG pathway analysis, mouse genes were converted to human orthologs as described in the materials and methods section. Hierarchical clustering of variables and box color of heat map was based on data normalization, mean $=0$, variance $=1$.

Table 1. Top inflammation-related enriched KEGG pathways using top 500 DEGs from CapEC.

\begin{tabular}{|c|c|c|c|c|c|}
\hline KEGG Pathway Term & $\begin{array}{l}\text { Gene } \\
\text { Count }\end{array}$ & $p$-Value & Fold Enrichment & Bonferroni & FDR \\
\hline mmu04668: TNF signaling pathway & 22 & $4.5 \times 10^{-12}$ & 6.8 & $1.0 \times 10^{-9}$ & $8.5 \times 10^{-10}$ \\
\hline $\begin{array}{l}\text { mmu04621: NOD-like receptor } \\
\text { signaling pathway }\end{array}$ & 10 & $3.7 \times 10^{-5}$ & 5.9 & $8.4 \times 10^{-3}$ & $1.7 \times 10^{-3}$ \\
\hline $\begin{array}{l}\text { mmu04620: Toll-like receptor signaling } \\
\text { pathway }\end{array}$ & 13 & $3.8 \times 10^{-5}$ & 4.3 & $8.5 \times 10^{-3}$ & $1.7 \times 10^{-3}$ \\
\hline mmu04210: Apoptosis & 10 & $5.7 \times 10^{-5}$ & 5.6 & 0.012 & $2.1 \times 10^{-3}$ \\
\hline $\begin{array}{l}\text { mmu04064: NF-kappa B signaling } \\
\text { pathway }\end{array}$ & 12 & $1.2 \times 10^{-4}$ & 4.1 & 0.027 & $3.3 \times 10^{-3}$ \\
\hline $\begin{array}{l}\text { mmu04060: Cytokine-cytokine receptor } \\
\text { interaction }\end{array}$ & 18 & $7.8 \times 10^{-4}$ & 2.5 & 0.161 & 0.011 \\
\hline mmu04010: MAPK signaling pathway & 17 & $3.0 \times 10^{-3}$ & 2.2 & 0.49 & 0.028 \\
\hline mmu04115: p53 signaling pathway & 8 & $3.4 \times 10^{-3}$ & 4.0 & 0.53 & 0.028 \\
\hline $\begin{array}{l}\text { mmu04350: TGF-beta signaling } \\
\text { pathway }\end{array}$ & 9 & $3.4 \times 10^{-3}$ & 3.5 & 0.54 & 0.028 \\
\hline $\begin{array}{l}\text { mmu04062: Chemokine signaling } \\
\text { pathway }\end{array}$ & 14 & $5.2 \times 10^{-3}$ & 2.4 & 0.68 & 0.037 \\
\hline
\end{tabular}


Table 2. Top inflammation-related enriched KEGG pathways using top 500 DEGs from PCV.

\begin{tabular}{|c|c|c|c|c|c|}
\hline KEGG Pathway Term & Gene Count & $p$-Value & Fold Enrichment & Bonferroni & FDR \\
\hline mmu04668: TNF signaling pathway & 20 & $1.1 \times 10^{-10}$ & 6.5 & $2.5 \times 10^{-8}$ & $2.27 \times 10^{-8}$ \\
\hline $\begin{array}{l}\text { mmu04064: NF-kappa B signaling } \\
\text { pathway }\end{array}$ & 14 & $2.5 \times 10^{-6}$ & 5.1 & $5.6 \times 10^{-4}$ & $1.2 \times 10^{-4}$ \\
\hline $\begin{array}{l}\text { mmu04620: Toll-like receptor } \\
\text { signaling pathway }\end{array}$ & 14 & $4.1 \times 10^{-6}$ & 4.9 & $8.8 \times 10^{-4}$ & $1.5 \times 10^{-4}$ \\
\hline $\begin{array}{l}\text { mmu04621: NOD-like receptor } \\
\text { signaling pathway }\end{array}$ & 10 & $2.4 \times 10^{-5}$ & 6.2 & $5.3 \times 10^{-3}$ & $6.7 \times 10^{-4}$ \\
\hline $\begin{array}{l}\text { mmu04060: Cytokine-cytokine } \\
\text { receptor interaction }\end{array}$ & 20 & $4.3 \times 10^{-5}$ & 2.9 & $9.3 \times 10^{-3}$ & $1.0 \times 10^{-3}$ \\
\hline mmu04210: Apoptosis & 8 & $1.3 \times 10^{-3}$ & 4.7 & 0.249 & 0.016 \\
\hline $\begin{array}{l}\text { mmu04514: Cell adhesion } \\
\text { molecules (CAMs) }\end{array}$ & 13 & $1.8 \times 10^{-3}$ & 2.8 & 0.335 & 0.022 \\
\hline $\begin{array}{l}\text { mmu04630: JAK-STAT signaling } \\
\text { pathway }\end{array}$ & 11 & $7.3 \times 10^{-3}$ & 2.7 & 0.794 & 0.065 \\
\hline $\begin{array}{l}\text { mmu04066: HIF-1 signaling } \\
\text { pathway }\end{array}$ & 9 & $7.5 \times 10^{-3}$ & 3.1 & 0.805 & 0.065 \\
\hline $\begin{array}{l}\text { mmu04350: TGF-beta signaling } \\
\text { pathway }\end{array}$ & 8 & $9.4 \times 10^{-3}$ & 3.3 & 0.871 & 0.072 \\
\hline
\end{tabular}

Our analysis also revealed that several transcription factors, such as, Rel (also known as $c$ Rel), Relb, Cebpb, Cebpd, Nfkbia, Nfkbie and Myc, were significantly upregulated in both capEC and PCV (Supplementary Materials S1 and S2). By use of Qlucore software 3.6 (33) (Qlucore AB, Lund, Sweden) we also predicted some transcription factor targets (TFTs) those found to regulate many DEGs ( $p \leq 0.05$, difference of at least 2-fold) both in capEC and PCV. Our findings suggest that transcription factor such as Crel, NF-kB65, Znf791, Cebpb and miR-649 might regulate most DEGs of capEC during sepsis (Table 3). For example, Crel was found to regulate genes involved in inflammation: Rel, Relb, Birc3, Bcl3; regulation of transcription: Nfkbia, Rel, Relb, Stat6, Tcf7l2; response to cytokine: Rel, Relb; regulation of focal assembly: S100a10, Sdc4; regulation of apoptosis: Nfkbia, Birc3, Ier3, Map3k8, Ube2d3. In PCV, we found that transcription factors such as Cebpb, Gata6 and several miRs such as miR-4769-3p, miR-362-5p, miR-510, might regulate most DEGs of PCV during sepsis (Table 2). For example, Gata6 was found to regulate genes involved in transcription process: Bcl6, Ctcf, Gpbp1, Lhx6, Smad5, Fosl2, Sall2; nodal signaling pathway: Tgif1, Tgif2; Atypical chemokine receptor: Ackr1 that acts as chemokine receptor for Ccl2, Ccl5, Ccl7, Ccl11, Cxcl5, Cxcl6, Il8 (human)/Cxcl2 (mouse); regulation of ubiquitination: Mid1 (E3 ubiquitin-protein ligase midline-1) that regulates innate immune response via NF-kB signaling.

\subsection{Validation by $q R T-P C R$}

To identify possible technical artifacts in our RNAseq data and to investigate whether the tissue processing for single-cell preparation have any impact on gene expression, we performed qRT-PCR of six randomly selected genes (Icam1, Pim1, Enc1, Selp, Mid1 and Il6) in freshly collected lung samples. The qPCR results showed that all selected genes consistently changed in the lung samples of CLP animals (Figure 7). 
Table 3. Top 5 transcriptional factor target (TFT) gene sets in lung capEC and PCV.

\begin{tabular}{|c|c|}
\hline GSEA Name of TFT & DEGs $(p<0.05)$ \\
\hline \multicolumn{2}{|l|}{ capEC } \\
\hline GSEA_CREL_01 & $\begin{array}{c}\text { BCL3, BIRC3, CASKIN1, CDC42SE1, CLDN5, CLOCK, COL11A2, CTDSP1, CYP2D6, FUT7, } \\
\text { GPBP1, IER3, IER5, ITPKC, MAP3K8, MIDEAS, MOB3C, NFKBIA, PIK3R3, RAP2C, } \\
\text { RBMS1, REL, RELB, S100A10, SDC4, STAT6, TACC1, TCF7L2, UBE2D3, UPF2, YWHAQ, } \\
\text { YWHAZ, ZFAND3, ZNF593 }\end{array}$ \\
\hline GSEA_NFKAPPAB65_01 & $\begin{array}{l}\text { BCL3, BIRC3, CDC42SE1, CLDN5, CLOCK, COL11A2, CTDSP1, CYP2D6, FGF17, FUT7, } \\
\text { GPBP1, HSP9OB1, IER3, IER5, ILK, ITPKC, MAP3K8, MIDEAS, MOB3C, NFKBIA, NFKBIB, } \\
\text { RAP2C, RBMS1, REL, RELB, RND1, SDC4, STAT6, UBE2D3, UPF2, YWHAQ, YWHAZ }\end{array}$ \\
\hline GSEA_ZNF791_TARGET_GENES & ADA, BRWD1, CWC25, CYP2D6, FES, GIT2, RND1, SDC4, STAT6, ZNF827 \\
\hline GSEA_TTGCWCAAY_CEBPB_02 & BNC2, CEBPB, HOXA5, KLF5, MIDEAS, NFKBIE, NRP2, RHOB, SLC7A11, TRIB1, VCAN \\
\hline MIR649 & $\begin{array}{c}\text { ACER3, BICRAL, CACNA1H, CCDC6, GAPVD1, INPP5K, KPNA1, MEX3C, MOB1B, } \\
\text { NUFIP2, RFX7, SENP5, STT3A, ZNF281 }\end{array}$ \\
\hline \multicolumn{2}{|l|}{ PCV } \\
\hline GSEA_GATA6_01 & $\begin{array}{c}\text { ACKR1, BCL6, CTCF, ECHDC2, ERRFI1, FBRS, FOSL2, GPBP1, IGFBP5, KIZ, LHX6, LIMS1, } \\
\text { LIX1, MID1, NOCT, NRAS, PCDH9, PFKFB1, PLAGL2, PSD4, SALL2, SLC39A14, SMAD5, } \\
\text { TACC1, TGIF1, TGIF2, TPH2, UBE2H, WAPL, XPO6 }\end{array}$ \\
\hline GSEA_TTGCWCAAY_CEBPB_02 & CSDE1, DBH, GSR, IDH1, IP6K2, MIDEAS, NFKBIE, RHOB, TRIB1, VCAN \\
\hline GSEA_MIR4769_3P & BCL6, CCDC6, ERBIN, NFKBIE, PAK2, PTEN, WAPL \\
\hline GSEA_MIR362_5P & $\begin{array}{c}\text { ATF1, CPEB4, EDEM1, IDH1, LARP1B, PEG10, PLAGL2, RBM27, RPS6KB1, SNX18, SYNC, } \\
\text { ADAM9, ATF1 }\end{array}$ \\
\hline GSEA_CCTGAGT_MIR510 & CAPZA2, IP6K2, WAPL, ZHX2 \\
\hline
\end{tabular}

A

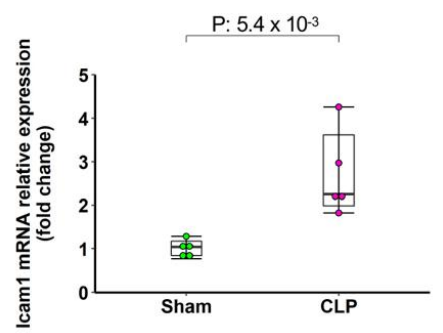

B

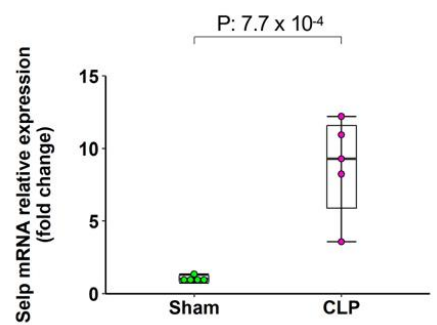

C

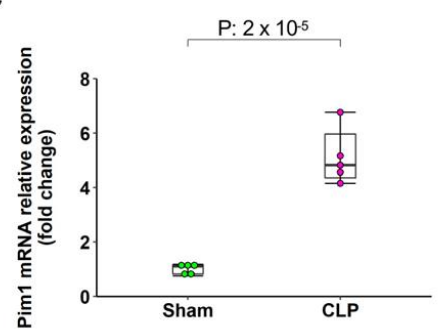

D

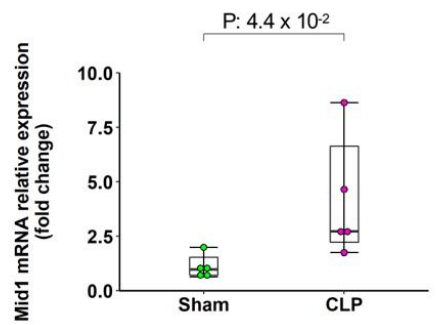

E

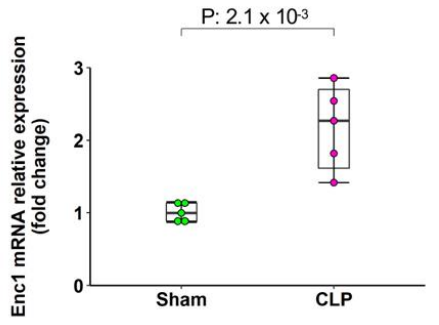

$\mathrm{F}$

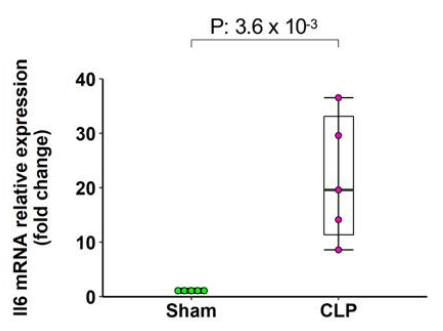

Figure 7. Validation of selected RNA-seq data by qRT-PCR. Samples were collected $4 \mathrm{~h}$ after induction of abdominal sepsis. Relative expressions of (A) Icam1, (B) Selp, (C) Pim1, (D) Mid1, (E) Enc1 and (F) Il6 mRNAs were quantified using qRT-PCR where GAPDH was used as a housekeeping gene for mRNA and expressions were determined using $2^{-\Delta \Delta C T}$ method. Box plots represent the median (25-75 percentile) and the whiskers extend from the minimum to the maximum levels and dots represent sample values and $n=5$. 


\section{Discussion}

Despite significant investigational efforts, sepsis remains a major health-care concern with a high mortality rate in intensive care units [20]. The treatment of sepsis is largely supportive apart from antibiotic therapies. Several promising anti-inflammatory and anticoagulant drugs have failed to improve septic survival in clinical trials $[5,14,21]$. The reason could be that sepsis turns itself into a multiple process making the therapeutic process difficult to treat with a single target. Hence, in the present study, we investigated global expressional changes in lung EC subsets during sepsis for the first time. We identified several groups of DEGs and pathways in capEC and PCV in sepsis, including genes involved in innate immune response, regulation of vascular permeability, regulation of coagulation, respiratory gaseous exchange, EC apoptosis, cell chemotaxis, cellular junction organization, protein transport and relevant signaling pathways. We also identified several transcription factors and miRNAs which could be targeted to reduce global inflammatory and metabolic changes in sepsis.

Our analysis revealed that sorted sham lung capEC and PCV expressed distinct signature gene sets. We observed that lung capEC are highly enriched with Cd36, Hey1, Notch4 and progenitor marker, such as, Nes, while in PCV, we observed high expression of CD63, Bst1, Tlr4 and Nr2f2 genes. These observations are in line with two other studies showing that in lymphoid tissue capEC and venous high EC express distinct signature genes $[17,19]$. We found that capEC are enriched with genes involved in specialized functions, such as, vasculogenesis: Acvrl1, Cd34; artery morphogenesis: Hey1, NPRL3; plasma membrane transport: Rab31, Rsc1a1; and regulation of blood circulation: Apln, Casr, Fga. In contrast, PCV was enriched with genes involved in leukocyte trafficking: Bst1, Vcam1, Jam1; and cytokine secretion process: Il33, Il6, Lbp. However, both capEC and PCV were highly enriched with genes related to coagulation: Cd36, Fga, Fibp, Prkce; and Anxa5, Gata6, Prkg1, Vwf, Sh2B2, respectively. Based on the differences we observed in sham capEC and PCV, we anticipated that the responses would be different during sepsis.

Lung microvessels are not only involved in transportation of nutrients and oxygen into tissues, but also actively involved in interactions with circulating cells (i.e., leukocytes, platelets, etc.), as well as secretion of mediators into the circulation and local tissues [14]. Our analysis revealed that sepsis caused upregulation or downregulation of the set of genes involved in regulation of membrane permeability, gaseous exchange, cellular junction, cytokine production, leukocyte migration, cell apoptosis, actin polymerization and protein transport across plasma membrane. A hallmark of sepsis is exudation of fluid and proteins from the intravascular space into the extravascular space of the lung. Experimental sepsis studies suggest that loss of fluid from blood vessels is caused by the breakdown of EC barrier function [22,23] and/or direct EC injury [24]. In the present study, we observed significant upregulation of genes, such as, Bnip3, naif1, Tb53bp1 and Ywhaz, and downregulation of genes, such as Casp8, Spg7, Acaa2, Hip1r and Ywhaq, in capEC related to vascular permeability. Notably, Bnip3 (Bcl2 interacting protein 3) has been shown to regulate human microvascular EC death [25] and overexpression of Naif1 (Nuclear apoptosis-inducing factor 1) has been reported to increase apoptosis of cancer cells [26]. Taken together, these studies support our finding that both Bnip3 and Naif1 are involved in EC death and permeability in sepsis. From the downregulated gene sets, we found downregulation of Acaa2 (Acetyl-CoA acyltransferase 2), which is known to inhibit mammary epithelial cell apoptosis [27], thus downregulation of Acaa2 during sepsis may increase apoptosis of capEC. EC permeability was related to other genes, such as, Cldn5 (claudin 5) and Pecam-1/CD31 (platelet EC adhesion molecule), also significantly upregulated in septic capEC, suggesting that sepsis induces gene expression related to capillary permeability. Although the function of other differentially expressed genes are not investigated in sepsis yet, it could be hypothesized that they all act in concurrence to damage microvessels during sepsis. Increase in protein and water transport during sepsis could also occur due to increase in transcellular transport and disruption of adherens junction [28]. In fact, in our data, we have observed downregulation of genes related to 
both adherens junction organization (Smad7, Nectin7, Dlg5, Add1, ctnnd1, Efnb2) and genes related to regulation of protein transport (App, Dmn2, Ano6, Kcnk6, Slmap). In addition, a large number of genes related to actin polymerization were changed during sepsis in the present study. This notion is supported by the fact that actin polymerization is a key regulator of EC gap formation and permeability during inflammation [29]. We also observed differential expression of genes related to Notch, Wnt and Hippo signaling. These observations are also in line with other studies showing that all these signaling pathways are involved in sepsis-induced lung inflammation [30-32]. Similar to capEC of lymph nodes [19], lung capEC in this study is also found to be featured with proangiogenic genes. Hence, sepsis caused downregulation of proangiogenic genes such as Vegfa, Nrarp, Jcad and upregulation of the anti-angiogenic gene Thbs1, indicating that the proliferative function of capEC might be disrupted in sepsis.

Moreover, circulatory monocytes and endothelial tissue play a key role in regulating blood coagulation and hemostasis [33-35]. We found that CLP caused upregulation of genes, such as Prkcd, Nfe2l2, Fcer1g and Syk and downregulation of genes, such as Edn1, Ano6 and Tepan8, which are closely linked to the coagulation cascade. Most of these genes, involved in coagulation, have not been examined in models of sepsis previously. Furthermore, plasminogen activator inhibitor-1 (Pai-1) is known to regulate fibrinolysis by inhibiting tissue plasminogen activator (tPA) and an increase in Pai-1 is associated with fibrin deposition in the lung [36]. In our data, we observed an increase in Serpine1 (gene responsible for Pai-1 production), suggesting that profibrinolytic properties of capEC is also disrupted and coagulation cascade might be activated, during sepsis. Although gene enrichment analysis revealed that response of capEC in terms of coagulation is higher than PCV, PCV also expressed a significant number of coagulation related genes. For example, we observed a significant increase in genes encoding F2rl2, F5, Thbs1, Serpind1 in lung PCV, suggesting that both PCV and capEC are important for coagulation cascade activation in sepsis.

Moreover, activation and dysfunction of microvascular EC occur as a result of exposure to pathogens and inflammatory mediators, as well as by adherent leukocytes and platelets [37,38]. In response to pathogens or damage associated molecular patterns (DMAPs), EC secrete cytokines and chemokines [39]. We found upregulation of Cxcl2 (macrophage inflammatory protein 2, Mip-2), Tnfrsf1b (Tnf Receptor Superfamily Member 1B), Ccl3 (Mip-1-alpha), Ccl4 (Mip-1ß), Il6 (interleukin 6) and Il12 (interleukin 12) in septic PCV. In addition, receptor for Il1, IL1 $r$ was also highly upregulated in septic PCV in our study. Furthermore, we observed that $C x c 12$ (stromal cell-derived factor 1) was downregulated in septic capEC.

Accumulating studies have shown that expression of cell adhesion molecules on EC and subsequent adhesion and migration of leukocytes via EC play a central role in septic-induced tissue injury [40-42]. Importantly, several experimental sepsis studies demonstrated that neutrophil infiltration through postcapillary venules is a rate limiting step [42-44]. In our study we observed significant upregulation of genes on the lung EC related to leukocyte migration. For example, genes related to leukocyte adhesion to EC, such as Selp (P-selectin), Icam1 (intercellular adhesion molecule 1), were significantly upregulated in PCV during sepsis. In addition, we observed upregulation of Sele (Eselectin) in our data (not in the top 500). Activation of these selectins on endothelium has previously been shown to facilitate leukocyte and platelets binding via their surface glycoproteins $[45,46]$. We also observed significant enrichment of glycosylation pathway in PCV which is known to regulate leukocyte recruitment and inflammation [47]. Moreover, similar to other studies [48,49], we found a significant upregulation of Icam1 in capEC, suggesting that activated leukocytes might bind with Icam1 and plug into capEC and cause EC damage.

We identified several transcription factor targets of our DEGs, of which NF- $\mathrm{kB}$ transcription factor plays a pivotal role in modulation of immune response during sepsis [50]. Accumulating data suggest that homo- and heterodimers of NF- $\mathrm{kB}$ transcription factors, 
such as, p65, RelA, RelB, cRel, p50, p52, p65, play a major role in regulating genes involved in aggravated inflammatory responses, including synthesis of cytokines, coagulation factors and cell adhesion molecules [51,52]. An oligonucleotide decoy of transcription factor, NF- $k B$, has been shown to reduce acute lung injury in CLP-induced mouse model of sepsis [53]. Targeting transcription factors using small molecules is known to be difficult because of complicated protein-protein and/or protein-DNA interactions. Interestingly, recent progress in peptide-based drug targets has been shown to accomplish such challenges to some extent $[54,55]$. However, it should be noted that targeting a transcription factor that regulates many biological processes may simultaneously disrupt other critical physiological responses, and further research is needed before clinical use.

The spectrum of EC dysfunction during sepsis is so complex that a detailed presentation of all aspects of changes is not possible in this paper. Nevertheless, we tried to discuss important molecular changes covering, EC barrier dysfunction, activation of coagulation system in relation to EC, leukocyte-EC interactions in relation to lung failure. In addition to confirming genes previously identified in sepsis, we identified several novel genes and candidate transcription factors of potential importance in sepsis. The functional role of previously unaddressed genes as well as the possibility of using some of these genes as early markers of lung damage and targeting of candidate transcription factors should be subjects of future experimental and clinical studies.

\section{Materials and Methods}

\subsection{Animal Experiments}

All experiments were conducted in accordance with the legislation on the protection of animals and approved by the Regional Ethical Committee for Animal Experimentation at Lund University, Lund, Sweden (Permit number: 5.8.18-08769/2019). Male C57BL/6 mice (20-25 g) were obtained from Zanvier labs, Le Genest-Saint-Isle, France, and kept in a pathogen-free facility on a 12-12 h light-dark cycle with free access to food and tap water. Mice were housed at least one week to acclimate to the new surroundings. Animals were anesthetized with $75 \mathrm{mg}$ of ketamine hydrochloride (Hoffman-La Roche, Basel, Switzerland) and 25 mg of xylazine (Janssen Pharmaceutical, Beerse, Belgium) per $\mathrm{kg}$ body weight. The ARRIVE guidelines were followed for all animal experiments [56]. Experimental lethal grade sepsis was induced by cecal ligation and puncture (CLP) as described elsewhere [57], and this model of sepsis is known to cause massive infiltration of neutrophils and lung injury [58,59]. Briefly, cecum was exposed, and feces were pushed towards the ascending colon. A ligature was made below the ileocecal valve covering $70 \%$ of the cecum, thereafter the cecum was punctured twice with a 21-gauge needle. Mice were returned to their cages and reanesthetized for sample collection $4 \mathrm{~h}$ after CLP procedure. For RNA-sequencing fresh samples were digested for single-cell suspension and lung EC were sorted out for total RNA extraction as described below. In separate experiment, blood and lung tissues were collected for biochemical and immunoassays as described below.

\subsection{Endothelial Subsets Sorting and Total RNA Extraction}

For EC subsets isolation, lung tissues were taken from the mice and digested to single-cell suspension. Briefly, tissues were minced in ice-cold HBSS and washed by centrifugation. Tissue pellets were then digested in HBSS media containing $0.2 \mathrm{mg} / \mathrm{mL}$ collagenase $\mathrm{P}, 0.8 \mathrm{mg} / \mathrm{mL}$ dispase II, $0.01 \mathrm{mg} / \mathrm{mL}$ DNase for $60 \mathrm{~min}$ at $37^{\circ} \mathrm{C}$ with gentle rocking. Dissociated cell suspensions were collected in FBS to stop digestion then passed through $100 \mu \mathrm{m}$ filter followed by $40 \mu \mathrm{m}$ filter. ECs were enriched from the resulting cell suspensions by depletion of hematolymphoid cells and epithelial cells using anti-CD45 and anti-CD326 microbeads (Miltenyi Biotech, Bergisch Gladbach, Germany). Enriched ECs were labelled with antibodies for FACS sorting. Briefly, anti-CD31 was used as the primary defining EC marker and anti-gp38 to distinguish between lymphatic (gp38+CD31+) and blood (gp38-CD31+) EC. A pool of lineage markers (anti-CD45, -CD11a, -TER119, -EpCAM) and 7-AAD were used to exclude hematolymphoid, epithelial, stromal cells and dead 
cells from the analysis. CapEC subset was identified as CD31+Icam1+Vcam1- and PCV subset was identified as CD31+Icam1+Vcam1+. EC subsets were sorted directly into RLT buffer (Qiagen, West Sussex, UK). Total RNA was isolated from the sorted cells with RNeasy Plus Micro kit (Qiagen) and the quality of RNA was checked by Bioanalyzer RNA 6000 pico assay (Agilent, Santa Clara, CA, USA). Cell isolation, purification and sorting were conducted in the presence of RNase inhibitor (Sigma-Aldrich, Stockholm, Sweden) throughout the protocol.

\subsection{RNA Sequencing and Data Analysis}

For whole-transcriptome analysis, sequencing was performed at the Center for Translational Genomics (CTG), Lund University and Clinical Genomics Lund, SciLifeLab. Briefly, sequence library was prepared using SMARTer ${ }^{\circledR}$ Stranded Total RNA-Seq Kit v2-Pico Input Mammalian (634411, Takara Bio USA Inc., Mountain View, CA, USA. After library preparation and quality check, the double-stranded-cDNA was sequenced on NextSeq 500 (SY-415-1001, Illumina) using (read one-index reads-read two, bp): 75-8-8-75 setup. The sequencing data was analyzed using the following pipeline: 'Demultiplexing' using the bcl2fastq2 software, 'Raw Quality Control' (QC) using FastQC, 'Read Mapping and $\mathrm{QC}^{\prime}$ was performed using HISAT2 software, and the reference genome sequence was from the Ensemble database, the Mouse 38, and the annotation (GTF) was from the release 93, 'Expression Counts' was performed using the StringTie software and finally 'Differentially Expressed Genes (DEGs)' analysis was performed using DESeq2 in the statistical environment $\mathrm{R}$ (version 4.0.2). Variables with three or more samples having zero counts were filtered out before analysis. Volcano plots and heatmaps using DEGs were generated using Qlucore Omics Explorer software version 3.6 (33) (Qlucore AB, Lund, Sweden). Data were normalized to 2-logarithm and threshold was set to +1 . Hierarchical clustering of variables and box color of heat maps was generated after data normalization to mean $=0$, variance $=1$.

\subsection{Flow Cytometry}

In a separate experiment, identity of capEC and PCV in enriched EC were confirmed by using additional capillary and venular markers. In one panel, anti-Icam1 and anti-CD63 antibodies were used to separate capEC and PCV from CD31+gp38- blood ECs (BECs) and in another panel anti-Ly6c and anti-Vcam1 antibodies were used to separate CD31+gp38BECs. For Mac1 expression on blood neutrophils, blood was collected from the inferior vena cava in acid citrate dextrose (1:10) at $4 \mathrm{~h}$ after CLP induction. Cells were incubated with an anti-CD16/CD32 for 10 min to block Fc $\gamma$ III/IIRs and reduce non-specific binding. Then, cells were incubated with a phycoerythrin-conjugated anti-Ly6g (clone 1A8, BD Pharmingen, San Jose, CA, USA) antibody and an allophycocyanin (APC)-conjugated antiMac-1 (clone M1/70, integrin $\alpha \mathrm{M}$ china, rat IgG2b, eBioscience Inc., San Diego, CA, USA) antibody at $4{ }^{\circ} \mathrm{C}$ for $20 \mathrm{~min}$. Red blood cells were lysed and neutrophils were recovered following centrifugation. Flow cytometric analysis was conducted using Cytoflex flow cytometer (Becton Dickinson, Mountain View, CA, USA). Mac-1 expression (MFI, mean fluorescence intensity) was determined on gated Ly6g positive cells.

\subsection{Myeloperoxidase (MPO) Assay}

Polymorphonuclear leukocytes (PMNLs) are known to contain higher amounts of MPO than mononuclear leukocytes (MNLs) and frequently used as an indicator of PMNLs accumulation in the tissue $[60,61]$. For MPO assay, lungs were perfused with $10 \mathrm{~mL}$ PBS first and several pieces of lung tissue were snap frozen and stored at $-80{ }^{\circ} \mathrm{C}$ for later assay. Frozen lung tissues were thawed and weighed and then homogenized in $1 \mathrm{~mL}$ mixture (4:1) of PBS and aprotinin (10,000 kallikrein inactivator units per ml; Trasylo, Bayer HealthCare AG, Leverkusen, Germany) for one minute. Homogenates were centrifuged for $10 \mathrm{~min}$ $\left(15,300 \times g, 4{ }^{\circ} \mathrm{C}\right)$, and supernatants were frozen to $-20{ }^{\circ} \mathrm{C}$, and stored for other analysis. $\mathrm{MPO}$ activity was determined in the remaining pellets in accordance with an established 
protocol [60]. Pellets were resuspended in 0.2 M PB pH7.4, then centrifuged, the pellets were again suspended in one $\mathrm{ml}$ of $0.5 \%$ hexadecyl-trimethylammonium bromide buffer. The dissolved samples were frozen for $24 \mathrm{~h}$, thawed, sonicated for $90 \mathrm{sec}$ and put in a water bath $\left(60^{\circ} \mathrm{C}, 2 \mathrm{~h}\right)$. Enzyme reaction with substrate $\left(3,3^{\prime}, 5,5^{\prime}\right.$-Tetramethylbenzidine, TMB) (Sigma Aldrich, Stockholm, Sweden) as measured spectrophotometrically (450 nm, with a reference filter $540 \mathrm{~nm}, 25^{\circ} \mathrm{C}$ ). Data were expressed as MPO units per gram tissue.

\subsection{ELISA}

Plasma and lung levels of $\mathrm{Il6}$ and Cxcl2 were quantified using double-antibody enzyme-linked immunosorbent assay kits (R\&D Systems Europe, Ltd., Abingdon, Oxon, UK). For plasma, blood was collected from the inferior vena cava in acid citrate dextrose solution, and then centrifuged $\left(2000 \times g\right.$ for $10 \min$ at $\left.4{ }^{\circ} \mathrm{C}\right)$. After centrifugation, plasma samples were collected and stored at $-20^{\circ} \mathrm{C}$ for ELISA. For measurement of 116 and Cxcl2 in lung tissue, lungs were perfused with $10 \mathrm{~mL}$ PBS first and several pieces of lung tissues were collected and snap frozen for later assay.

\subsection{Gene Sets Enrichment Analysis and KEGG Pathway Analysis}

In order to reveal and visualize biological processes involved in the pathogenesis of abdominal sepsis, we used Qlucore Omics Explorer software version 3.6 (33) (Qlucore $\mathrm{AB}$, Lund, Sweden). For GSEA analysis, mouse gene symbols (first letter in upper case) were converted to human orthologs (all letters in upper case) using Ensembl databases by BioMart databases [62] in Bioconductor (version 3.12). GSEA analysis was performed using human orthologs which have corresponding mouse gene symbols. C5 gene ontology collection derived from the Molecular Signatures Database (MSigDB v7.2, Broad Institute, Cambridge, MA, USA) [63] was used for GSEA. Analysis was performed with a ranking metric based on two group comparisons. Qlucore GSEA displays Running Enrichment Score (RES), the match positions for the genes in the selected data set and the ranking metric values for the input gene list.

Kyoto Encyclopedia of Genes and Genomes (KEGG) pathway enrichment analyses were performed using the top 500 DEGs from capEC and PCV in Database for Annotation, Visualization and Integration Discovery (DAVID version 6.7; http://david.abcc.ncifcrf. gov) (accessed date: 26 March 2021) [64,65]. GO biological process terms were based on biological process of Molecular Signatures Database (v7.2 MSigDB). Mouse gene symbols were converted to human orthologs using Ensembl databases as described above and then used in DAVID. Hierarchical clustering of variables and box color of heat maps were generated after data normalization to mean $=0$, variance $=1$ in Qlucore program.

\subsection{Validation by $q R T-P C R$}

For the validation of RNA sequencing data, total RNA was extracted from freshly collected lung tissue by using TRIzol (Invitrogen, Thermo Fisher Scientific, Inc., Waltham, MA, USA) and purified using Direct-zol RNA extraction kit (Zymo Research, Irvine, CA, USA) according to manufacturer's recommendations. cDNA was synthesized using 0.5 $\mu \mathrm{g}$ total RNA using RevertAid First Strand cDNA Synthesis kit (Thermo Fisher Scientific $^{\mathrm{TM}}$, Milford, MA, USA). Real-time polymerase chain reaction (RT-PCR) was performed using TB Green Advantage qPCR Premix (Clontech, Mountain View, CA, USA) in MX 3000P detection system (Stratagene, AH diagnostics, Stockholm, Sweden). Expression of target genes relative to GAPDH were determined using $2^{-\Delta \Delta C T}$ method. Experimentally validated PCR primers from PrimerBank (https://pga.mgh.harvard.edu/primerbank/) (accessed date: 15 June 2021) were used for validation (Table 4). 
Table 4. List of primers for validation by qRT-PCR.

\begin{tabular}{|c|c|c|c|c|c|}
\hline \multicolumn{2}{|c|}{ Mouse Gene Name } & \multirow{2}{*}{$\begin{array}{c}\text { Primer Sequence }\left(\mathbf{5}^{\prime}->\mathbf{3}^{\prime}\right) \\
\text { GTGATGCTCAGGTATCCATCCA }\end{array}$} & \multirow{2}{*}{$\begin{array}{c}\text { Length } \\
22\end{array}$} & \multirow{2}{*}{$\begin{array}{c}\operatorname{Tm} \\
61.3\end{array}$} & \multirow{2}{*}{$\begin{array}{c}\text { Location } \\
74-95\end{array}$} \\
\hline Icam1 & Forward Primer & & & & \\
\hline & Reverse Primer & CACAGTTCTCAAAGCACAGCG & 21 & 62.0 & $286-266$ \\
\hline \multirow[t]{2}{*}{ Mid1 } & Forward Primer & CTGTGACGGCACCTGTCTC & 19 & 62.3 & $1293-1311$ \\
\hline & Reverse Primer & AAACGGCTGACTGTTGGTCTT & 21 & 62.2 & $1497-1477$ \\
\hline \multirow[t]{2}{*}{ Pim1 } & Forward Primer & CTGGAGTCGCAGTACCAGG & 19 & 61.4 & $100-118$ \\
\hline & Reverse Primer & CAGTTCTCCCCAATCGGAAATC & 22 & 60.7 & 240-219 \\
\hline \multirow[t]{2}{*}{ Enc1 } & Forward Primer & CTGTTTCATAAGTCСТCCTACGC & 23 & 60.4 & $64-86$ \\
\hline & Reverse Primer & CACCACTGAACATGGCTTCG & 20 & 61.3 & $232-213$ \\
\hline \multirow[t]{2}{*}{ Il6 } & Forward Primer & CCAAGAGGTGAGTGCTTCCC & 20 & 62.2 & $462-481$ \\
\hline & Reverse Primer & CTGTTGTTCAGACTCTCTCCСT & 22 & 60.8 & $579-558$ \\
\hline \multirow[t]{2}{*}{ Selp } & Forward Primer & CATCTGGTTCAGTGCTTTGATCT & 23 & 60.5 & $69-91$ \\
\hline & Reverse Primer & ACCCGTGAGTTATTCCATGAGT & 22 & 60.8 & $173-152$ \\
\hline
\end{tabular}

\subsection{Statistical Analysis}

Statistical analysis of RNA-seq data was performed using DESeq2 in the statistical environment of $\mathrm{R}$ (version 4.0.2) and visualization was conducted using either Qlucore Omics Explorer (version 3.6 (33)) or R program. Box plots represent the median (25-75 percentile) and the whiskers extend from the minimum to the maximum levels. Dots represent sample expression values normalized to mean $=0$, variance $=1$ for RNAseq data or sample values for other experiments. Significant differences between two groups were evaluated using the student's $t$-test. $p<0.05$ was considered statistically significant and for RNAseq data $\mathrm{p}$ adjusted (padj) value indicates $p$ value after correction using Bonferroni method. $n$ represents number of animals or experiments per group.

\section{Conclusions}

Our results show that sepsis caused massive transcriptional changes and that these changes differ in capEC and PCV. We believe that beyond the discussion addressed here, our data will provide extensive resources for discovery of additional genes and pathways involved in other inflammatory lung diseases. In conclusion, through transcriptomic analysis of lung capEC and PCV, we have identified several new genes and possible pathways involved in leukocyte recruitment, dysfunction of endothelium and disruption of homeostasis. We propose that targeting some of the identified pathways or transcription factors regulating the above-discussed functions could be beneficial to ameliorate sepsisinduced organ damage in experimental and clinical settings.

Supplementary Materials: The following are available online at https:/ /www.mdpi.com/article/10 .3390/ijms221910181/s1.

Author Contributions: Conceptualization, H.T. and M.R.; methodology, M.R. and Z.D.; software, M.R. and Z.D.; validation, M.R., Z.D. and C.-F.R.; formal analysis, M.R. and Z.D.; investigation, resources, data curation, M.R. and Z.D.; writing—original draft preparation, M.R. and H.T.; writingreview and editing, M.R., Z.D., C.-F.R. and H.T.; visualization, M.R. and Z.D.; supervision, H.T.; project administration, M.R.; funding acquisition, H.T and M.R. All authors have read and agreed to the published version of the manuscript.

Funding: This work was supported by funding from Swedish Research Council (2017-01621), Maggie Stephens foundation and Einar and Inga Nilsson foundation. Zhiyi Ding was supported by grants from the China Scholarship Council (201706310181). 
Institutional Review Board Statement: All experiments were conducted in accordance with the legislation on the protection of animals and approved by the Regional Ethical Committee for Animal Experimentation at Lund University, Sweden (Permit number: 5.8.18-08769/2019).

Informed Consent Statement: Not applicable.

Data Availability Statement: RNAseq data are deposited at Gene Expression Omnibus (GEO) database of NCBI (National Center for Biotechnology Information). (GEO Accession: GSE169532).

Acknowledgments: RNA sequencing was performed at the Center for Translational Genomics (CTG), Lund University, and Clinical Genomics Lund, SciLifelab. We thank Cecilia Wahlström and Srinivas Veerla of CTG for their technical support.

Conflicts of Interest: The authors declare no conflict of interest.

\section{References}

1. Singer, M.; Deutschman, C.S.; Seymour, C.W.; Shankar-Hari, M.; Annane, D.; Bauer, M.; Bellomo, R.; Bernard, G.R.; Chiche, J.D.; Coopersmith, C.M.; et al. The Third International Consensus Definitions for Sepsis and Septic Shock (Sepsis-3). JAMA 2016, 315, 801-810. [CrossRef] [PubMed]

2. Robson, W.P.; Daniel, R. The Sepsis Six: Helping patients to survive sepsis. Br. J. Nurs. 2008, 17, 16-21. [CrossRef] [PubMed]

3. Rudd, K.E.; Johnson, S.C.; Agesa, K.M.; Shackelford, K.A.; Tsoi, D.; Kievlan, D.R.; Colombara, D.V.; Ikuta, K.S.; Kissoon, N.; Finfer, S.; et al. Global, regional, and national sepsis incidence and mortality, 1990-2017: Analysis for the Global Burden of Disease Study. Lancet 2020, 395, 200-211. [CrossRef]

4. Cohen, J. The immunopathogenesis of sepsis. Nature 2002, 420, 885-891. [CrossRef]

5. Ince, C.; Mayeux, P.R.; Nguyen, T.; Gomez, H.; Kellum, J.A.; Ospina-Tascon, G.A.; Hernandez, G.; Murray, P.; De Backer, D.; Workgroup, A.X. The Endothelium in Sepsis. Shock 2016, 45, 259-270. [CrossRef]

6. Joffre, J.; Hellman, J.; Ince, C.; Ait-Oufella, H. Endothelial Responses in Sepsis. Am. J. Respir. Crit. Care Med. 2020, 202, 361-370. [CrossRef]

7. Vassiliou, A.G.; Kotanidou, A.; Dimopoulou, I.; Orfanos, S.E. Endothelial Damage in Acute Respiratory Distress Syndrome. Int. J. Mol. Sci. 2020, 21, 8793. [CrossRef]

8. Ploppa, A.; Schmidt, V.; Hientz, A.; Reutershan, J.; Haeberle, H.A.; Nohe, B. Mechanisms of leukocyte distribution during sepsis: An experimental study on the interdependence of cell activation, shear stress and endothelial injury. Crit. Care 2010, 14, R201. [CrossRef]

9. Wang, Y.; Roller, J.; Menger, M.D.; Thorlacius, H. Sepsis-induced leukocyte adhesion in the pulmonary microvasculature in vivo is mediated by CD11a and CD11b. Eur. J. Pharmacol. 2013, 702, 135-141. [CrossRef] [PubMed]

10. Liu, L.; Kubes, P. Molecular mechanisms of leukocyte recruitment: Organ-specific mechanisms of action. Thromb. Haemost. 2003, $89,213-220$.

11. Scarlatescu, E.; Juffermans, N.P.; Thachil, J. The current status of viscoelastic testing in septic coagulopathy. Thromb. Res. 2019, 183, 146-152. [CrossRef]

12. Lupu, F.; Kinasewitz, G.; Dormer, K. The role of endothelial shear stress on haemodynamics, inflammation, coagulation and glycocalyx during sepsis. J. Cell Mol. Med. 2020, 24, 12258-12271. [CrossRef]

13. Aird, W.C. The role of the endothelium in severe sepsis and multiple organ dysfunction syndrome. Blood 2003, 101, 3765-3777. [CrossRef]

14. Lehr, H.A.; Bittinger, F.; Kirkpatrick, C.J. Microcirculatory dysfunction in sepsis: A pathogenetic basis for therapy? J. Pathol. 2000, 190, 373-386. [CrossRef]

15. Pries, A.R.; Secomb, T.W. Modeling structural adaptation of microcirculation. Microcirculation 2008, 15, 753-764. [CrossRef]

16. Friederici, H.H. On the diaphragm across fenestrae of capillary endothelium. J. Ultrastruct. Res. 1969, 27, 373-375. [CrossRef]

17. Lee, M.; Kiefel, H.; LaJevic, M.D.; Macauley, M.S.; Kawashima, H.; O'Hara, E.; Pan, J.; Paulson, J.C.; Butcher, E.C. Transcriptional programs of lymphoid tissue capillary and high endothelium reveal control mechanisms for lymphocyte homing. Nat. Immunol. 2014, 15, 982-995. [CrossRef] [PubMed]

18. Doyle, E.L.; Ridger, V.; Ferraro, F.; Turmaine, M.; Saftig, P.; Cutler, D.F. CD63 is an essential cofactor to leukocyte recruitment by endothelial P-selectin. Blood 2011, 118, 4265-4273. [CrossRef]

19. Brulois, K.; Rajaraman, A.; Szade, A.; Nordling, S.; Bogoslowski, A.; Dermadi, D.; Rahman, M.; Kiefel, H.; O'Hara, E.; Koning, J.J.; et al. A molecular map of murine lymph node blood vascular endothelium at single cell resolution. Nat. Commun. 2020, 11, 3798. [CrossRef] [PubMed]

20. Vincent, J.L.; Jones, G.; David, S.; Olariu, E.; Cadwell, K.K. Frequency and mortality of septic shock in Europe and North America: A systematic review and meta-analysis. Crit. Care 2019, 23, 196. [CrossRef]

21. Freeman, B.D.; Natanson, C. Anti-inflammatory therapies in sepsis and septic shock. Expert Opin. Investig. Drugs 2000, 9, 1651-1663. [CrossRef] 
22. Ni, J.; Lin, M.; Jin, Y.; Li, J.; Guo, Y.; Zhou, J.; Hong, G.; Zhao, G.; Lu, Z. Gas6 Attenuates Sepsis-Induced Tight Junction Injury and Vascular Endothelial Hyperpermeability via the Axl/NF-kappaB Signaling Pathway. Front. Pharmacol. 2019, 10, 662. [CrossRef] [PubMed]

23. Schmidt, T.T.; Tauseef, M.; Yue, L.; Bonini, M.G.; Gothert, J.; Shen, T.L.; Guan, J.L.; Predescu, S.; Sadikot, R.; Mehta, D. Conditional deletion of FAK in mice endothelium disrupts lung vascular barrier function due to destabilization of RhoA and Rac1 activities. Am. J. Physiol. Lung Cell Mol. Physiol. 2013, 305, L291-L300. [CrossRef]

24. Cheng, K.T.; Xiong, S.; Ye, Z.; Hong, Z.; Di, A.; Tsang, K.M.; Gao, X.; An, S.; Mittal, M.; Vogel, S.M.; et al. Caspase-11-mediated endothelial pyroptosis underlies endotoxemia-induced lung injury. J. Clin. Invest. 2017, 127, 4124-4135. [CrossRef]

25. Jurasz, P.; Yurkova, N.; Kirshenbaum, L.; Stewart, D.J. VEGF masks BNIP3-mediated apoptosis of hypoxic endothelial cells. Angiogenesis 2011, 14, 199-207. [CrossRef]

26. Lv, B.; Shi, T.; Wang, X.; Song, Q.; Zhang, Y.; Shen, Y.; Ma, D.; Lou, Y. Overexpression of the novel human gene, nuclear apoptosis-inducing factor 1, induces apoptosis. Int. J. Biochem. Cell Biol. 2006, 38, 671-683. [CrossRef]

27. Yang, Y.; Fang, X.; Yang, R.; Yu, H.; Jiang, P.; Sun, B.; Zhao, Z. MiR-152 Regulates Apoptosis and Triglyceride Production in MECs via Targeting ACAA2 and HSD17B12 Genes. Sci. Rep. 2018, 8, 417. [CrossRef] [PubMed]

28. Komarova, Y.; Malik, A.B. Regulation of endothelial permeability via paracellular and transcellular transport pathways. Annu. Rev. Physiol. 2010, 72, 463-493. [CrossRef]

29. Vouret-Craviari, V.; Boquet, P.; Pouyssegur, J.; Van Obberghen-Schilling, E. Regulation of the actin cytoskeleton by thrombin in human endothelial cells: Role of Rho proteins in endothelial barrier function. Mol. Biol. Cell 1998, 9, 2639-2653. [CrossRef]

30. Xia, S.; Menden, H.L.; Korfhagen, T.R.; Kume, T.; Sampath, V. Endothelial immune activation programmes cell-fate decisions and angiogenesis by inducing angiogenesis regulator DLL4 through TLR4-ERK-FOXC2 signalling. J. Physiol. 2018, 596, 1397-1417. [CrossRef] [PubMed]

31. Sharma, A.; Yang, W.L.; Ochani, M.; Wang, P. Mitigation of sepsis-induced inflammatory responses and organ injury through targeting Wnt/beta-catenin signaling. Sci. Rep. 2017, 7, 9235. [CrossRef] [PubMed]

32. Lv, Y.; Kim, K.; Sheng, Y.; Cho, J.; Qian, Z.; Zhao, Y.Y.; Hu, G.; Pan, D.; Malik, A.B.; Hu, G. YAP Controls Endothelial Activation and Vascular Inflammation Through TRAF6. Circ. Res. 2018, 123, 43-56. [CrossRef] [PubMed]

33. Chen, X.; Lao, Y.; Yi, J.; Yang, J.; He, S.; Chen, Y. SENP3 in monocytes/macrophages up-regulates tissue factor and mediates lipopolysaccharide-induced acute lung injury by enhancing JNK phosphorylation. J. Cell Mol. Med. 2020, 24, 5454-5462. [CrossRef] [PubMed]

34. Okamoto, K.; Tamura, T.; Sawatsubashi, Y. Sepsis and disseminated intravascular coagulation. J. Intensive Care 2016, 4, 23. [CrossRef] [PubMed]

35. Vallet, B. Bench-to-bedside review: Endothelial cell dysfunction in severe sepsis: A role in organ dysfunction? Crit Care 2003, 7, 130-138. [CrossRef]

36. Whyte, C.S.; Morrow, G.B.; Mitchell, J.L.; Chowdary, P.; Mutch, N.J. Fibrinolytic abnormalities in acute respiratory distress syndrome (ARDS) and versatility of thrombolytic drugs to treat COVID-19. J. Thromb. Haemost. 2020, 18, 1548-1555. [CrossRef]

37. Lehr, H.A.; Arfors, K.E. Mechanisms of tissue damage by leukocytes. Curr. Opin. Hematol. 1994, 1, 92-99. [PubMed]

38. Bozza, F.A.; Shah, A.M.; Weyrich, A.S.; Zimmerman, G.A. Amicus or adversary: Platelets in lung biology, acute injury, and inflammation. Am. J. Respir. Cell Mol. Biol. 2009, 40, 123-134. [CrossRef]

39. Mantovani, A.; Bussolino, F.; Dejana, E. Cytokine regulation of endothelial cell function. FASEB J. 1992, 6, 2591-2599. [CrossRef]

40. Zhao, Y.J.; Yi, W.J.; Wan, X.J.; Wang, J.; Tao, T.Z.; Li, J.B.; Wang, J.F.; Deng, X.M. Blockade of ICAM-1 improves the outcome of polymicrobial sepsis via modulating neutrophil migration and reversing immunosuppression. Mediators Inflamm. 2014, 2014, 195290. [CrossRef]

41. Croner, R.S.; Lehmann, T.G.; Fallsehr, C.; Herfarth, C.; Klar, E.; Kirschfink, M. C1-inhibitor reduces hepatic leukocyte-endothelial interaction and the expression of VCAM-1 in LPS-induced sepsis in the rat. Microvasc. Res. 2004, 67, 182-191. [CrossRef]

42. Herter, J.M.; Rossaint, J.; Spieker, T.; Zarbock, A. Adhesion molecules involved in neutrophil recruitment during sepsis-induced acute kidney injury. J. Innate Immun. 2014, 6, 597-606. [CrossRef]

43. Nolte, D.; Kuebler, W.M.; Muller, W.A.; Wolff, K.D.; Messmer, K. Attenuation of leukocyte sequestration by selective blockade of PECAM-1 or VCAM-1 in murine endotoxemia. Eur. Surg. Res. 2004, 36, 331-337. [CrossRef]

44. Matsukawa, A.; Lukacs, N.W.; Hogaboam, C.M.; Knibbs, R.N.; Bullard, D.C.; Kunkel, S.L.; Stoolman, L.M. Mice genetically lacking endothelial selectins are resistant to the lethality in septic peritonitis. Exp. Mol. Pathol. 2002, 72, 68-76. [CrossRef]

45. Vestweber, D. How leukocytes cross the vascular endothelium. Nat. Rev. Immunol. 2015, 15, 692-704. [CrossRef] [PubMed]

46. Coenen, D.M.; Mastenbroek, T.G.; Cosemans, J. Platelet interaction with activated endothelium: Mechanistic insights from microfluidics. Blood 2017, 130, 2819-2828. [CrossRef] [PubMed]

47. Lowe, J.B. Glycan-dependent leukocyte adhesion and recruitment in inflammation. Curr. Opin. Cell Biol. 2003, 15, 531-538. [CrossRef]

48. Aoki, T.; Suzuki, Y.; Nishio, K.; Suzuki, K.; Miyata, A.; Iigou, Y.; Serizawa, H.; Tsumura, H.; Ishimura, Y.; Suematsu, M.; et al. Role of CD18-ICAM-1 in the entrapment of stimulated leukocytes in alveolar capillaries of perfused rat lungs. Am. J. Physiol. 1997, 273, H2361-H2371. [CrossRef]

49. Miranda, M.; Balarini, M.; Caixeta, D.; Bouskela, E. Microcirculatory dysfunction in sepsis: Pathophysiology, clinical monitoring, and potential therapies. Am. J. Physiol. Heart Circ. Physiol. 2016, 311, H24-H35. [CrossRef] 
50. Brown, M.A.; Jones, W.K. NF-kappaB action in sepsis: The innate immune system and the heart. Front. Biosci. 2004, 9, $1201-1217$. [CrossRef] [PubMed]

51. Oeckinghaus, A.; Ghosh, S. The NF-kappaB family of transcription factors and its regulation. Cold Spring Harb. Perspect. Biol. 2009, 1, a000034. [CrossRef]

52. Mussbacher, M.; Salzmann, M.; Brostjan, C.; Hoesel, B.; Schoergenhofer, C.; Datler, H.; Hohensinner, P.; Basilio, J.; Petzelbauer, P.; Assinger, A.; et al. Cell Type-Specific Roles of NF-kappaB Linking Inflammation and Thrombosis. Front. Immunol. 2019, 10, 85. [CrossRef]

53. Matsuda, N.; Hattori, Y.; Jesmin, S.; Gando, S. Nuclear factor-kappaB decoy oligodeoxynucleotides prevent acute lung injury in mice with cecal ligation and puncture-induced sepsis. Mol. Pharmacol. 2005, 67, 1018-1025. [CrossRef] [PubMed]

54. Mylonis, I.; Chachami, G.; Simos, G. Specific Inhibition of HIF Activity: Can Peptides Lead the Way? Cancers 2021, 13, 410. [CrossRef]

55. Inamoto, I.; Shin, J.A. Peptide therapeutics that directly target transcription factors. Pept. Sci. 2019, 111, e24048. [CrossRef]

56. Du Sert, N.; Hurst, V.; Ahluwalia, A.; Alam, S.; Avey, M.T.; Baker, M.; Browne, W.J.; Clark, A.; Cuthill, I.C.; Dirnagl, U.; et al. The ARRIVE guidelines 2.0: Updated guidelines for reporting animal research. PLoS Biol. 2020, 18, e3000410.

57. Rittirsch, D.; Huber-Lang, M.S.; Flierl, M.A.; Ward, P.A. Immunodesign of experimental sepsis by cecal ligation and puncture. Nat. Protoc. 2009, 4, 31-36. [CrossRef] [PubMed]

58. Aziz, M.; Ode, Y.; Zhou, M.; Ochani, M.; Holodick, N.E.; Rothstein, T.L.; Wang, P. B-1a cells protect mice from sepsis-induced acute lung injury. Mol. Med. 2018, 24, 26. [CrossRef]

59. Asaduzzaman, M.; Lavasani, S.; Rahman, M.; Zhang, S.; Braun, O.O.; Jeppsson, B.; Thorlacius, H. Platelets support pulmonary recruitment of neutrophils in abdominal sepsis. Crit. Care Med. 2009, 37, 1389-1396. [CrossRef]

60. Schierwagen, C.; Bylund-Fellenius, A.C.; Lundberg, C. Improved method for quantification of tissue PMN accumulation measured by myeloperoxidase activity. J. Pharmacol. Methods 1990, 23, 179-186. [CrossRef]

61. Mullane, K.M.; Kraemer, R.; Smith, B. Myeloperoxidase activity as a quantitative assessment of neutrophil infiltration into ischemic myocardium. J. Pharmacol. Methods 1985, 14, 157-167. [CrossRef]

62. Durinck, S.; Spellman, P.T.; Birney, E.; Huber, W. Mapping identifiers for the integration of genomic datasets with the R/Bioconductor package biomaRt. Nat. Protoc. 2009, 4, 1184-1191. [CrossRef] [PubMed]

63. Subramanian, A.; Tamayo, P.; Mootha, V.K.; Mukherjee, S.; Ebert, B.L.; Gillette, M.A.; Paulovich, A.; Pomeroy, S.L.; Golub, T.R.; Lander, E.S.; et al. Gene set enrichment analysis: A knowledge-based approach for interpreting genome-wide expression profiles. Proc. Natl. Acad. Sci. USA 2005, 102, 15545-15550. [CrossRef] [PubMed]

64. Sherman, B.T.; Huang da, W.; Tan, Q.; Guo, Y.; Bour, S.; Liu, D.; Stephens, R.; Baseler, M.W.; Lane, H.C.; Lempicki, R.A. DAVID Knowledgebase: A gene-centered database integrating heterogeneous gene annotation resources to facilitate high-throughput gene functional analysis. BMC Bioinform. 2007, 8, 426. [CrossRef] [PubMed]

65. Kanehisa, M. Molecular network analysis of diseases and drugs in KEGG. Methods Mol. Biol. 2013, 939, $263-275$. 